\title{
Assessing the viability of silicate nanoclusters as carriers of the anomalous microwave emission: a quantum mechanical study
}

\author{
A. Macià Escatllar ${ }^{1}$ and S. T. Bromley ${ }^{1,2}$
}

\author{
${ }^{1}$ Departament de Ciència de Materials i Química Fisica Institut de Química Teòrica i Computacional, Universitat de Barcelona, \\ C/Martí i Franquès 1, 08028 Barcelona, Spain \\ 2 Institució Catalana de Recerca i Estudis Avançats (ICREA), 08010 Barcelona, Spain \\ e-mail: s.bromley@ub.edu
}

Received 31 July 2019 / Accepted 10 January 2020

\begin{abstract}
Nanosized silicate dust is likely to be abundant in many astronomical environments and it is a prime candidate for being the source of the anomalous microwave emission (AME). To assess the viability of silicate nanoclusters as AME carriers, their detailed properties need to be established. Using quantum chemical calculations, we compute the accurate chemical and electronic structures of three families of nanoclusters with astrophysically relevant compositions: $\mathrm{Mg}$-rich olivine $\left(\mathrm{Mg}_{2} \mathrm{SiO}_{4}\right)_{N}, \mathrm{Mg}$-rich pyroxene $(\mathrm{MgSiO})_{N}$, and silicon monoxide $(\mathrm{SiO})_{N}$, all in the $\leq 1 \mathrm{~nm}$ diameter size regime and for neutral and \pm 1 charge states. From these fundamental data, we directly derive the shapes, ionization potentials, electron affinities, and dipole moments of all nanoclusters. The aspect ratio of the nanoclusters fluctuates significantly with $N$ for small sizes, but especially for the olivine and pyroxene nanoclusters, it tends to stabilize towards $\sim 1.3$ for the largest sizes considered. These latter two nanocluster families tend to have mass distributions consistent with approximately prolate ellipsoidal shapes. Our calculations reveal that the dipole moment of all our nanoclusters can be substantially affected by changes in chemical structure (i.e. different isomers for a fixed $N$ ), ionisation, and substitution of $\mathrm{Mg}$ by Fe. Although all these factors are important, the dipole moments of our Mg-rich nanoclusters are always found to be large enough to account for the observed AME. However, $(\mathrm{SiO})_{N}$ nanoclusters are only likely to be potential AME contributors when they are both charged and their chemical structures are anisotropically segregated. We also model the emissivity per $\mathrm{H}$ of a representative $\left(\mathrm{Mg}_{2} \mathrm{SiO}_{4}\right)_{3}$ nanocluster by directly calculating the quantum mechanical rotational energy levels and assuming a distribution of occupied levels in accordance with equilibrium Boltzmann statistics. We compare our bottom-up results with previously published classical models and show that a population of silicate nanoclusters containing only $1 \%$ of the total Si budget can reproduce the AME emissivity.
\end{abstract}

Key words. dust, extinction - ISM: lines and bands - radio continuum: ISM - radiation mechanisms: general - astrochemistry molecular data

\section{Introduction}

The anomalous microwave emission (AME) is detected as a foreground feature which typically peaks between 20 and $30 \mathrm{GHz}$ and extends over a 10-60 GHz range (Dickinson et al. 2018). The AME has been observed in many astrophysical environments including the diffuse ISM (Hildebrandt et al. 2007) and galactic clouds (Watson et al. 2005). It is also likely that AME is fundamentally related to the excess microwave emission observed in circumstellar environments (Ubach et al. 2017; Hoang et al. 2018; Greaves et al. 2018). Nanosized dust grains, hereafter referred to as nanoclusters, are expected to be the origin of the AME due to the spatial correlation of AME with regions of dust infrared (IR) emission (Ade et al. 2011; Casassus et al. 2006; Finkbeiner et al. 2002). In the case of circumstellar shells, a theoretical study by Rafikov (2006) showed that with our current understanding of emission mechanisms, the excess microwave emission observed in circumstellar shells should have a large contribution from spinning dust. Several mechanisms could, in principle, explain the AME depending on the type of grain that produces it. For example, iron-containing nanoclusters could contribute due to their magnetic properties (Draine \& Lazarian 1999), while silicate and carbonaceous nanoclusters could emit due to thermal emission (Agladze et al. 1996) and/or rapid spinning (Ali-Haïmoud et al. 2009; Draine \& Lazarian 1999, 1998; Erickson 1957; Jones 2009). A recent review of available observational evidence and astrophysical modelling concluded that spinning dipolar nanoclusters are the most likely cause of the AME (Dickinson et al. 2018). As the grain rotation rate must be rather high to account for the AME, studies have focused on nanoclusters with typical diameters of $\leq 1 \mathrm{~nm}$.

Initially, spinning polycyclic aromatic hydrocarbons (PAHs) were proposed as candidates for the source of the AME (Draine \& Lazarian 1998). However, it has since been shown that there is no correlation between the observed IR intensities of PAHs and AME intensities (Hensley et al. 2015), which implies that the AME likely originates from other nanograin carriers (Hensley et al. 2015; Tibbs et al. 2011; Vidal et al. 2011). Although large quantities of both silicate and carbonaceous dust can be verified through their characteristic IR spectra, as yet, there is no clear observational evidence to support any one particular type of nanograin as the general source of the AME. Recently, carbon nanodiamonds have been put forward as a possible source of AME in protoplanetary disks (Greaves et al. 2018). From a bottom-up chemical perspective, one potential problem with this proposal is the origin of the necessary dipoles in such species. As carbon is inherently a non-polar covalently bonded material, one must also provide evidence of 
an astrophysically feasible process via which a sufficiently high dipole is produced in such potential carriers. Recently, silicate nanoclusters have been proposed as possible general carriers of the AME (Hoang et al. 2016; Hensley \& Draine 2017). Here, as the chemical bonding in silicates is more ionic, nanosilicates will intrinsically tend to be polar without the need for invoking external processes for dipole creation.

Currently employed models of microwave emission from spinning nanoclusters allow any type of nanograin to contribute to the AME as long as it is sufficiently abundant and has a large enough dipole moment (Ali-Haïmoud 2013; Dickinson et al. 2018). The work of Draine \& Lazarian (1998) established the foundations on how to compute the rotational energy distribution from nanoclusters taking into account the effect of several processes such as grain-gas collisions, photon absorption/emission, $\mathrm{H}_{2}$ formation on the surface of the grain, interactions with the plasma, etc. Later models developed refinements such as including grain wobbling and precession (Hoang et al. 2010), testing the effect of asymmetric grains (Hoang et al. 2011) or using the Fokker-Planck equation to obtain more accurate rotational energy distributions (Ali-Haïmoud et al. 2009). All these approaches are based on detailed attempts to describe relevant (astro)physical phenomena and how they could affect rotational energy distributions. In such works, estimates of the magnitudes of nanograin dipole moments are based on largely qualitative top-down arguments (i.e. from general considerations of properties of bulk materials). The dipole moments derived by such means may be quite reasonable, however, the lack of a more quantitative and accurate basis for such estimates hinders our ability to test the validity of various potential nanograin sources of AME. In principle, however, the microwave emission from spinning nanoclusters can be derived directly in a more bottomup manner (i.e. from their detailed chemical/structural/electronic properties) and thus the potential contributions to the AME from different nanocluster types could be calculated and compared theoretically.

Ideally, to obtain the accurate dipole moment of a nanocluster, one requires its atomistically detailed chemical structure, the associated electronic charge distribution and its overall charge. In order to find stable chemical structures of nanoclusters one can employ global optimisation structure searches (Catlow et al. 2010). Further, by using quantum chemical modelling one can then derive the electronic structure of such nanoclusters and various astronomically relevant properties (e.g. dipole moments, IR spectra, heat capacities...). Recently, following such an approach, we reported low energy structures of silicate nanoclusters of both $\mathrm{Mg}$-rich olivine $\left(\mathrm{Mg}_{2} \mathrm{SiO}_{4}\right)_{N}$ and pyroxene $\left(\mathrm{MgSiO}_{3}\right)_{N}$ stoichiometries with $N=1-10$ (Escatllar et al. 2019), and $(\mathrm{SiO})_{N}$ nanoclusters with $N=1-20$ (Bromley et al. 2016). In all cases, the largest considered nanocluster size was of the order of $1 \mathrm{~nm}$ diameter. As judged by detections of characteristic IR bands at 10 and $18 \mu \mathrm{m}, \mathrm{Mg}$-rich silicate dust particles are commonly found in a wide range of astrophysical environments (Fogerty et al. 2016; Kessler-Silacci et al. 2006; Molster et al. 2002; Waelkens et al. 1996). Based only on this high abundance, silicate nanoclusters are a potential candidate source for the AME. Assuming that silicate nanoclusters contain $15 \%$ of the total Si budget in the ISM (Li \& Draine 2001), Hoang et al. (2016) estimated that silicate nanoclusters may be able to explain the entirety of the observed AME. Molecular $\mathrm{SiO}$ is also present in large quantities in the ISM, and its condensation into nanosized species in the cold environment of the ISM has been shown to be viable experimentally (Krasnokutski et al. 2014). As such, $(\mathrm{SiO})_{N}$ nanoclusters could also be considered as a possible contributor to the AME.
Herein, we derive accurate dipole moments $(\mu)$, ionization potentials (IP) and electron affinities (EA) directly from accurate quantum chemical calculations of stable nanoclusters of olivine $\left(\mathrm{Mg}_{2} \mathrm{SiO}_{4}\right)_{N}$, pyroxene $\left(\mathrm{MgSiO}_{3}\right)_{N}$ and $(\mathrm{SiO})_{N}$ silicon monoxide for neutral, and \pm 1 charge states and for sizes up to $1 \mathrm{~nm}$ in diameter. Where possible we compare our bottom-up derived data with previous reported top-down modelling studies. Although we show that some assumptions in the top-down derivation of nanosilicate properties are inaccurate, we confirm that nearly all our considered olivine and pyroxene nanoclusters do possess dipole moments with magnitudes that are sufficient to lead to significant contributions to the AME. For all our nanoclusters we provide the rotational constants and dipole moments in Appendix B.

To compute the emissivity of our nanoclusters, we employ an alternative approach to the one generally used in the literature. Herein, due to the fact that we have calculated the atomic and electronic structure of a range of stable silicate nanoclusters, we use this information to directly calculate the rotational energy levels in a bottom-up fashion using quantum mechanics. In this respect, our approach is similar to the one used for PAHs as reported by Ysard \& Verstraete (2010). In the present work, we apply statistical thermodynamics to compute the population of each rotational level. In effect, this approach assumes that all the physical processes described in the above works act together to bring the nanocluster rotational energy distribution to local thermodynamic equilibrium (LTE). As such, the current methodology should be most accurate in conditions where LTE is likely to be fulfilled, such as molecular clouds or dark clouds, and then only requires an effective rotational temperature. In a future work, we plan to consider non-equilibrium conditions for more accurately modelling other astrophysical environments.

Here we show that quantum chemical modelling can be a powerful and accurate tool for investigating detailed astrophysically relevant properties of silicate nanoclusters. Together with constraints from observation and laboratory studies, our bottom-up approach can complement more traditional top-down modelling approaches. In particular, we aim to provide more secure underpinnings for assessing the properties, abundance and astrophysical relevance of nanosized silicate dust.

\section{Methodology}

The detailed atomistic structures of all considered $\mathrm{Mg}$-pure Olivine $\left(\mathrm{Mg}_{2} \mathrm{SiO}_{4}\right)_{N}, \mathrm{Mg}$-pure Pyroxene $\left(\mathrm{MgSiO}_{3}\right)_{N}$ and silicon monoxide $(\mathrm{SiO})_{N}$ nanoclusters (hereafter referred to as O- $N, \mathrm{P}-N$ and $\mathrm{SiO}-N$, respectively) were obtained from extensive searches of low energy isomers as reported in Escatllar et al. (2019) and Bromley et al. (2016). In each case, the atomistic and electronic structure of all considered nanoclusters were optimised using all-electron density functional theory (DFT) based quantum chemical calculations using the hybrid PBE0 (Adamo \& Barone 1999) functional. The calculations were performed using the allelectron FHI-AIMS code (Blum et al. 2009) using a Tier1/light atom-centred numerical basis set which has an accuracy comparable to a TZVP Gaussian-type orbital basis set (Lamiel-Garcia et al. 2017).

From the masses of each atomic species and the optimized atomistic structures of the nanoclusters we can calculate the moments of inertia $I_{n}$ where $n \in x, y, z$ and where we take: $I_{z} \geq I_{y} \geq I_{x}$. The ratios between moments of inertia allow us to determine the approximate shape of the nanoclusters. Although the moments of inertia are rigorously defined, as we are dealing with species that contain only a few tens of atoms, the derived 
measure of nanograin spheroidal shape should be carefully interpreted as being associated with a mass-weighted spatial distribution of different atom types. If $I_{z}=I_{y}=I_{x}$, then $I_{n} / I_{x}=1$ and the mass distribution can be associated with a sphere. When $I_{z}>I_{y}$ and $I_{y} / I_{x}=1$, the nanocluster can be described by an oblate spheroid, with the degree of oblateness given by the magnitude of $I_{z} / I_{y}$. Alternatively, the case where $I_{y}>I_{x}$ and $I_{z}=I_{y}$ corresponds to a prolate spheroid, with the degree of prolateness given by the magnitude of $I_{y} / I_{x}$. In all other cases where the three moments of inertia are different the nanocluster shape can be classed as being between the two limiting oblate and prolate cases.

The dipole moment $(\mu)$ of each nanocluster was directly computed from its calculated electron density and nuclear positions. The full electron density $(\rho(\boldsymbol{r}))$ is readily obtained from DFT calculations, allowing to compute $\mu$ by:

$\boldsymbol{\mu}=\int \boldsymbol{r} \rho(\boldsymbol{r}) \mathrm{d} \boldsymbol{r}+\sum_{A} Z_{A} \boldsymbol{R}_{A}$,

where the left-hand integral term corresponds to the electron contribution to $\boldsymbol{\mu}$, and the right-hand term sums over all nuclei $\mathrm{A}$, with nuclear charge $\mathrm{Z}_{A}$ and position $\boldsymbol{R}_{A}$. Specifically, we report $\mu$ values for P- $N$ and O- $N$ for $N=1-10$ and SiO- $N$ for $N=2-$ 20 in their neutral state and \pm 1 charged states. The IP and EA values were also calculated for all neutral nanoclusters. The IP is defined as the energy required to extract an electron from the highest occupied molecular orbital (HOMO), therefore:

$\mathrm{IP}=\Delta E_{\mathrm{M} \rightarrow \mathrm{M}^{+}}=E\left(\mathrm{M}^{+}\right)-E(\mathrm{M})$,

where $E(\mathrm{M})$ is the total calculated energy of a neutral nanocluster, $\mathrm{M}$, and $E\left(\mathrm{M}^{+}\right)$is the energy of nanocluster, $\mathrm{M}$, with one electron removed. The EA is defined as the energy released upon attachment of a free-electron onto a nanocluster, with positive sign meaning a release in energy, and negative sign meaning that electron attachment requires energy (Zhan et al. 2003). Hence, it is calculated as:

$\mathrm{EA}=-\Delta E\left(\mathrm{M} \rightarrow \mathrm{M}^{-}\right)=E(\mathrm{M})-E\left(\mathrm{M}^{-}\right)$

where $E(\mathrm{M})$ is the total calculated energy of a neutral nanocluster, $\mathrm{M}$, and $E\left(\mathrm{M}^{-}\right)$is the energy of nanocluster, $\mathrm{M}$, with one electron added. This definition is consistent with that reported in Weingartner \& Draine (2001a). We note that the values reported in this work correspond to vertical EA and IP values (i.e. where the nanocluster structure in its charged and neutral state is unchanged).

The rotational spectrum of a nanocluster can be computed quantum mechanically by calculating the eigenvalues of the rotational Hamiltonian $\left(H_{r}\right)$. For symmetric nanoclusters, the angular momentum operators $\left(P^{2}\right.$ : square of the total angular momentum, $P_{Z}$ : space-fixed angular momentum projection on the $Z$-axis, and $P_{z}$ : body-fixed angular momentum projection on the $z$-axis) commute with $H_{r}$ and thus the energies can be found easily (Ysard \& Verstraete 2010). However, for asymmetric top species, such as most of the nanoclusters in this work, the $P_{z}$ operator does not commute with $H_{r}$. In such cases, the rotational wavefunction can be described as linear combinations of symmetric top wavefunctions, after which the rotational Hamiltonian is diagonalized. For a complete description of the calculation of such rotational spectra, we refer the reader to more detailed sources (Gordy \& Cook 1984). Here, we use the ASROT software (Kisiel 2001) from the PROSPE website (Kisiel 2019) to compute the frequencies $(v)$ in $\mathrm{MHz}$ and line intensities $(\mathcal{I})$ in $\mathrm{nm}^{2} \mathrm{MHz}$ at a given temperature $(T)$ of each allowed transition using the rotational constants $\left(B_{n}\right)$. From the two former quantities and using $T=300 \mathrm{~K}$, we can obtain the Einstein coefficient of spontaneous emission for the $i \rightarrow j$ transition $\left(A_{i j}\right)$ (Pickett et al. 1998) as:

$A_{j i} \approx \mathcal{I}_{j i}(T) v_{j i}\left[Q_{\mathrm{rs}}(T) / g^{\prime}\right] \mathrm{e}^{E^{\prime} / k T} \cdot 1.748 \times 10^{-9}$,

where $v_{i j}$ is the transition frequency between the states $i$ and $j$ in $\mathrm{MHz}, Q_{\mathrm{rs}}(T)$ is the rotational partition function at $T=300 \mathrm{~K}$, $g^{\prime}$ is the degeneracy of the upper-state $\left(g^{\prime}=2 J+1\right), E^{\prime}$ is the upper state energy in $\mathrm{cm}^{-1}, k$ is the Boltzmann constant and $1.748 \times 10^{-9}$ converts the resulting units to $s^{-1}$. $Q_{\mathrm{rs}}$ can be approximated as:

$Q_{\mathrm{rs}}=\frac{\pi^{1 / 2}}{\sigma_{r}} \frac{T^{3 / 2}}{\left(\Theta_{r, x} \Theta_{r, y} \Theta_{r, z}\right)^{1 / 2}}$,

where $\Theta_{r, n}$ as the rotational temperature for the $x, y$ or $z$ axis. The former approximation of $Q_{\mathrm{rs}}$ is valid as long as $\Theta_{r} / T$ is smaller than unity. For our nanoclusters, the values never exceed 0.001 even with $T=10 \mathrm{~K}$. As in the work of Ysard \& Verstraete (2010), the total power emitted by a specific transition is calculated as:

$P(j)=A_{j i} E_{j i} J / s$.

To obtain the power emitted in a given astronomical environment it is further required to add the population fraction of the upper state $j\left(\frac{\mathcal{N}_{j}}{\mathcal{N}_{g}}\right)$, the total number of nanoclusters in that environment $\left(\mathcal{N}_{g}\right)$ and divide by $4 \pi$ to obtain the power emitted per steradian:

$P(j)=A_{i j} \cdot v_{i j} \cdot h \cdot \frac{\mathcal{N}_{j}}{\mathcal{N}_{g}} \cdot \mathcal{N}_{g} \cdot \frac{1}{4 \pi} J / s s r^{-1}$,

where $h$ is the Planck constant. Finally, the emissivity is obtained by dividing the given $P$ by the number of hydrogen atoms $\left(n_{\mathrm{H}}\right)$ in the given environment $\left(\mathcal{J} / n_{\mathrm{H}}=P / n_{\mathrm{H}}\right)$. From this approach, the only free parameters are the number of nanoclusters, the fraction of nanoclusters in a given energy state $j$ and the number of hydrogen atoms. Most available models compute the population of each rotational level by a detailed description of the physics of each environment (Draine \& Lazarian 1998; Ali-Haïmoud et al. 2009; Ysard \& Verstraete 2010), including gas-grain collisions, plasma-drag, $\mathrm{H}_{2}$ formation on the surface of the nanoclusters, energy transfer between vibrational and rotational states and several other mechanisms. Gas-grain interactions and energy transfer between vibrational and rotational states should in principle allow reaching a stationary distribution of the rotational energy. We thus expect that in some dense environments, such as molecular clouds (MC), dark clouds (DC) and reflection nebulas $(\mathrm{RN})$, the populations of each rotational energy level can be approximated by statistical mechanics. The fraction of particles in the state $j$ can then be computed by:

$\frac{\mathcal{N}_{j}}{\mathcal{N}_{g}}=\frac{g^{\prime} \mathrm{e}^{-E^{\prime} / k T}}{Q_{r}(T)}$,

where $T$ is the rotational temperature of the grain. While the dust $T$ values in Draine \& Lazarian (1998) correspond to total grain temperatures, here we take them to be nanocluster rotational temperatures. In Appendix A we show how our results compare with results from Draine \& Lazarian (1998) and Ali-Haïmoud et al. (2015) for a fictitious asymmetric PAH with 
a number of atoms ( $N_{\text {atoms }}$ ) equal to 20 and $\mu=1.8 D$ in selected astronomical environments, confirming that rotational energy in thermal equilibrium can reproduce AME calculations.

The final parameter required is the number of silicate nanoclusters $\mathcal{N}_{g}$. The works of Weingartner \& Draine (2001b) and Li \& Draine (2001) provide the basis for most distributions employed in other theoretical studies of AME (Hoang et al. 2010; Draine \& Hensley 2016; Ali-Haïmoud et al. 2009). The work of Li \& Draine (2001) provides upper abundance limits of silicate nanoclusters with radii $\leq 1 \mathrm{~nm}$ to be $<15 \%$ of the total Si budget, based on extrapolated IR properties. The study of Weingartner \& Draine (2001b) provides a size distribution function capable of reproducing the observed extinction in the Milky Way and Magellanic Clouds. However, the work of Hensley \& Draine (2017) and Hoang et al. (2016) suggests that the fraction of small nanosilicate grains could be limited by microwave emission observations. For example, if silicate nanoclusters have large dipole moments, fewer nanoclusters would be needed to reproduce the observed emissivities. In this work, we tentatively explore this possibility by taking a representative nanosilicate containing 21 atoms with $\mathrm{Mg}_{2} \mathrm{SiO}_{4}$ composition (i.e. O-3) and computing the required abundance of such nanoclusters needed to qualitatively reproduce the results of other reported studies for an MC environment. The population of our representative nanosilicate grain is defined as the total $\mathrm{Si}$ atoms per $\mathrm{H}$ nucleon $\left(3.65 \times 10^{-5}\right)$ as in Weingartner \& Draine $(2001 \mathrm{~b})$, times the fraction of $\mathrm{Si}$ atoms contained in nanoclusters $\left(Y_{g r}\right)$, divided by the number of $\mathrm{Si}$ atoms in each nanocluster (3):

$\mathcal{N}_{g}=3.65 \times 10^{-5} \cdot Y_{g r} / 3$.

With our accurate chemical structures, the rotational constants $\mathrm{B}_{n}$ are directly available from:

$B_{n}=\frac{h}{8 \pi^{2} I_{n}}$,

where $I_{n}$ is the moment of inertia corresponding to the one of three orthogonal rotational axes $n \in x, y, z$. In Appendix B we report $B_{n}$ values for all our nanoclusters.

The spectra derived in this work correspond to rigid-rotor spectra under thermodynamic equilibrium and thus have some intrinsic bias towards longer wavelengths as we do not incorporate the effect of centrifugal distortion. The addition of centrifugal distortion terms is beyond the scope of the present paper as their calculation generally requires significant extra computational effort (Puzzarini et al. 2010). Nevertheless, to estimate the possible effect of distortion constants, we computed the distortion coefficients from anharmonic frequency calculations as described in Barone (2005) for the P-1 and P-2 nanoclusters using the Gaussian16 code (Frisch et al. 2016), employing DFT based calculations with the PBE0 functional and a $6-31 \mathrm{G}^{*}$ basis set. In Appendix B we provide an estimate of spectral change due to the inclusion of centrifugal distortion, which ranges from 0.2 to $3 \%$ with increasing size, from the $\mathrm{P}-1$ to $\mathrm{O}-10$ nanocluster, respectively.

\section{Nanocluster shape}

In Fig. $1 \mathrm{~A}$ and B, we plot $I_{y} / I_{x}$ versus $I_{z} / I_{x}$ for all considered nanocluster structures in order to provide an overview of their shape distribution. In principle, amorphous grains (i.e. with no long-range crystalline atomic order) should not have any preferred growth directions. As such, in the absence of external anisotropic factors, the intrinsic average shape of amorphous grains is expected to be spherical. In contrast, for example, crystalline grains tend to exhibit facetted morphologies and are thus intrinsically non-spherical (Zamirri et al. 2019). The nanocluster structures presented in our work are clearly not crystalline and hence it may be expected that they would become spherical as their size increases. Interestingly, we actually find that many of our silicate nanoclusters are still far away from being spherically symmetric for the size range we consider. In general, a large fraction of nanoclusters is found inside the prolate area of the plot. The largest $\mathrm{O}-\mathrm{N}$ nanoclusters are prolate with two moments of inertia $40 \%$ larger than the smallest one. On the other hand, the largest $\mathrm{P}-N$ nanoclusters are slightly oblate but relatively closer to the spherical limit. Conversely, the largest $\mathrm{SiO}$ nanoclusters are largely prolate. The elongation of $\mathrm{SiO}$ nanoclusters is a consequence of the side-by-side segregated nature of such species, containing silica-rich $\left(\mathrm{SiO}_{2}\right)$ and silicon-rich $(\mathrm{Si})$ fractions respectively, which causes an elongation of the nanocluster structure (Bromley et al. 2016).

In Fig. 1C and D we report the shapes of two large sets of silicate nanoclusters with fixed sizes and compositions but variable chemical structures. Specifically, we plot $I_{y} / I_{x}$ versus $I_{z} / I_{y}$ for a set of 180 P-6 isomers (C) and 180 O-6 isomers (D). In these plots, the colour code represents the relative energy of each isomer with respect to the lowest energy structure in each set. The random distribution of colours in both plots confirms the lack of correlation between energetics and shape. The P-6 structures cover a wide range of $I_{y} / I_{x}$ and $I_{z} / I_{x}$ values with both ratios reaching up to 3 and with respective median values of 1.51 and 1.78. For P-6 these two ratios are correlated with each other such that the plotted data follow a fairly narrow band that lays close and parallel with the limiting prolate shape line. Only for $I_{z} / I_{x}$ values lower than $\sim 1.6$ do we find a few P-6 structures that are dominantly oblate. Overall, only $20 \%$ of the particles appear to be below the line that separates the prolate from oblate particles.

The O-6 structures cover a smaller range of shape variability than the P-6 set with all $I_{y} / I_{x}$ and $I_{z} / I_{x}$ values being less than 2 and respective median values of 1.17 and 1.37. As in the P-6 set, the majority of the O-6 structures have shapes which tend to be more prolate. Here, however, the tendency is slightly less pronounced with the O-6 set, and the fraction of particles in the oblate region is $37 \%$.

Several studies have tried to obtain dust grain shapes by calculating the polarization caused by differently shaped particles and comparing it to observation. Most models rely on spheroidal particles, where the free parameter is the axial to equatorial ratio, also known as aspect ratio $(\mathrm{AR}=a / b)$ with $a>b$. Several works have reproduced observed polarizations using grains with AR values of 1.33 (Gupta et al. 2005) or between 1 and 2 (Draine \& Fraisse 2009). The largest AR values are reported by Das et al. (2010) to be between 2 and 3. Although these studies tend to address the shape of relatively large grains (i.e. size $\sim 0.1 \mu \mathrm{m}$ ), the evolution of AR values with increasing size from the nanocluster scale upwards can potentially help us understand the origin of the shape of larger grains. The ultrasmall nanoclusters considered herein can be thought of as nucleation seeds for dust growth, as considered in the works of Gobrecht et al. (2016) and Goumans \& Bromley (2012), and are thus likely to influence the shape of larger grains. In Fig. 2 we report the change in the AR values of our nanoclusters with respect to the number of atoms. For very small nanocluster sizes there is a high degree of fluctuation in the AR values for all considered nanocluster species. For the $\mathrm{SiO}-N$ nanoclusters this behaviour persists for all sizes. However, for the O- $N$ and $\mathrm{P}-N$ set of nanosilicates the AR values 

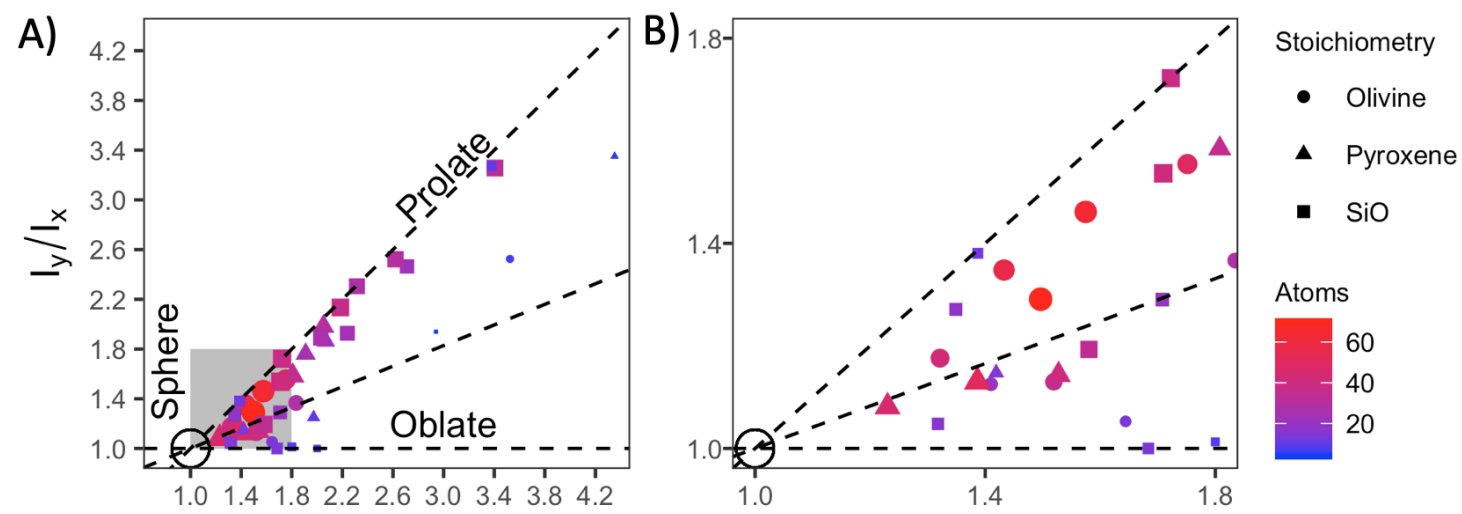

\section{C) \\ P-6 isomers}

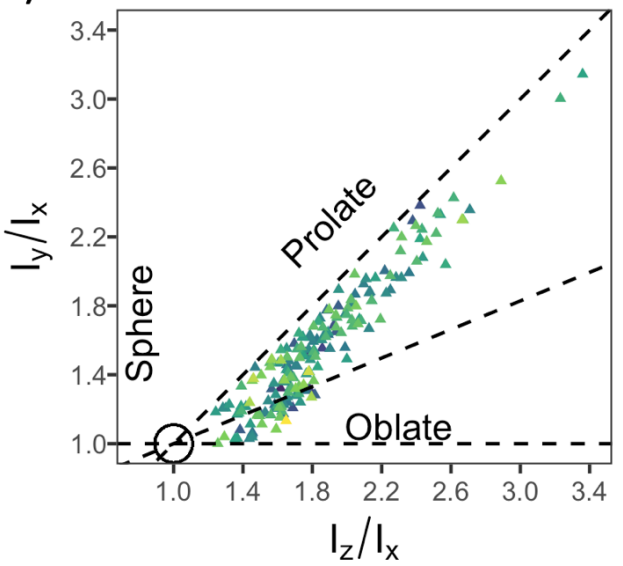

D) $0-6$ isomers

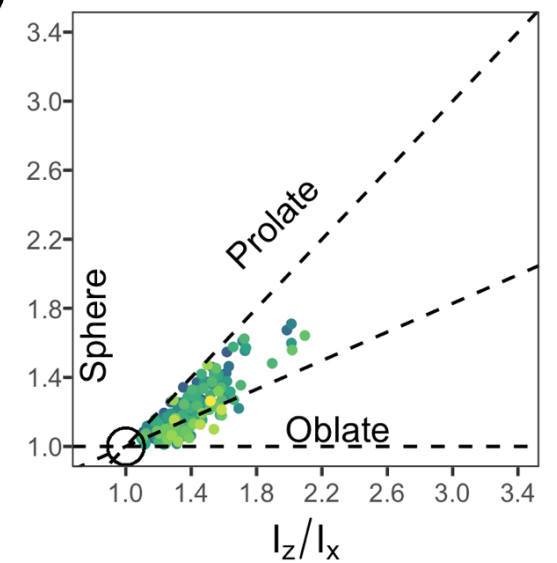

Rel. Energy / eV

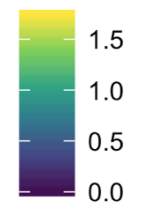

Fig. 1. Ratio between the largest and smallest moment of inertia $\left(I_{z} / I_{x}\right)$ with respect to the ratio between the intermediate moment of inertia to the smallest one $\left(I_{y} / I_{x}\right)$ for three selected sets of nanoclusters. The black circle indicates the ratio that defines a perfect sphere $\left(I_{y} / I_{x}=I_{z} / I_{x}=1\right)$, while the dashed lines show the prolate $\left(I_{z}=I_{y}>I_{x}\right)$ and oblate $\left(I_{x}=I_{y}<I_{z}\right)$ limits. (A) Lowest energy O-N and P-N nanoclusters for $N=1-10$ and SiO- $N$ nanoclusters for $N=1-20$, grey area corresponds to the plotted area in $(B)$. $(B)$ Zoom of the plot in $(A)$ over a 1.0-1.8 range for both axes. (C) 180 P-6 nanocluster isomers. $(D)$ 180 O-6 nanocluster isomers.

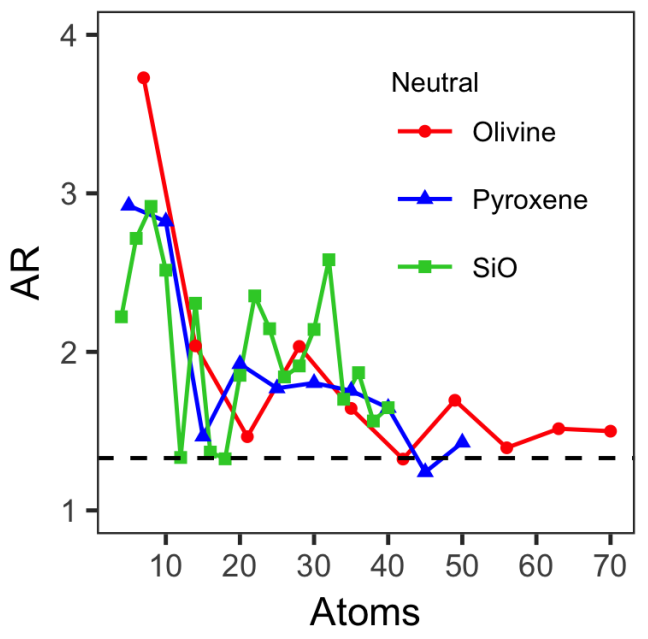

Fig. 2. Aspect ratio (AR) of O- $N, \mathrm{P}-N$ and $\mathrm{SiO}-N$ nanoclusters with respect to the number of atoms in each nanocluster. The dashed line corresponds to $\mathrm{AR}=1.33$ as determined by Gupta et al. (2005).

seem to be stabilizing at larger sizes to lay in a range between 1.7 and 1.3 in line with the values used by Gupta et al. (2005) and Draine \& Fraisse (2009). We note that these results are tentative as they correspond to a tendency derived from only a few nanoclusters over a small size range.

\section{Dipole moments}

The proposal for nanosilicates being the source of the AME was established in the works of Hoang et al. (2016) and Hensley \& Draine (2017). Using an expected fraction of silicate nanoclusters relative to the total $\mathrm{Si}$ abundance in the ISM of between 0.06 and 0.14 , they concluded that nanosilicates could explain the entirety of the observed AME in the ISM. In order to reach this conclusion it was necessary that the silicate nanoclusters should comply with:

$\beta=\mu / \sqrt{N_{\text {atoms }}} \geq 0.3$,

where $N$ is the number of atoms in the nanocluster and $\mu$ is the dipole moment in Debyes. Without detailed accurate data pertaining to such nanosilicates, the compliance with the above criterion was justified by the following hypothesis: since the $\mathrm{Si}-\mathrm{O}$ bond is polar and the structure of the nanosilicates is assumed to be amorphous, random orientations of the $\mathrm{Si}-\mathrm{O}$ bonds will lead to the formation of a permanent dipole in the nanosilicate. Assuming a simple near-spherical volume dependence on $N_{\text {atoms }}$ (using a bulk silicate density) and a fixed value for the dipole moment of the $\mathrm{Si}-\mathrm{O}$ bond, a random walk can be used to estimate values of $\mu$ for different values of $N_{\text {atoms }}$ (Hoang et al. 2016).

In the present work, the dipole moments of our nanoclusters are calculated directly from their charge distributions (see above). In Fig. 3 we show the chemical structures and charge 

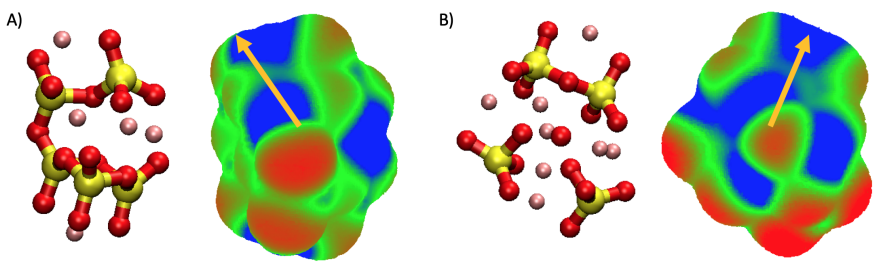

Fig. 3. Chemical structure (left) and electrostatic potential projected upon the electron density (right) for most stable $\left(\mathrm{MgSiO}_{3}\right)_{5} \mathrm{P}-5$ nanocluster isomer $(A)$, and the most stable $\left(\mathrm{Mg}_{2} \mathrm{SiO}_{4}\right)_{4} \mathrm{O}-4$ nanocluster isomer $(B)$. Atom colour key: $\mathrm{Si}-$ yellow, $\mathrm{O}-$ red, $\mathrm{Mg}-$ pink. The charge density isosurface value is $0.1 \mathrm{e}^{\mathrm{A}} \AA^{3}$. The electrostatic potential ranges from -0.07 (blue) to 0.07 (red) in $\mathrm{AU}$. The orange arrows indicate the direction of the dipole moment vector.

density distributions for the P-5 and O-4 nanoclusters. Here, the charge density is bounded by a surface which follows a fixed charge density value (i.e. a charge density isosurface) typical for the outer region of an atom. Such isosurfaces provide a visual means to appreciate the detailed atomistic shape of a nanocluster. The colouring of the isosurfaces is proportional to the electrostatic potential at each point (i.e. a measure of the energy required to take an electron from zero electric field to a point on the isosurface). The blue (negative) regions correspond to regions for which it is more energetically favourable to place an electron (i.e. indicating local excess positive charge), and red (positive) regions correspond to where it is more energetically costly to place an electron (indicating local excess negative charge). Green regions correspond to more neutral areas. Overall, the presence of a dipole in a nanocluster is compatible with spatial asymmetries of the electrostatic potential on an isosurface which surrounds the nanocluster. Clearly, in the cases of P-5 and O-4, the blue and red areas do not symmetrically cover the isosurfaces of each nanocluster and thus a dipole is expected in both cases. The direction of the dipole in each case is shown by an arrow.

We note that the Si cations always sit centrally with respect to four tetrahedrally bonded $\mathrm{O}$ anions and are thus always inside the nanoclusters. Perfectly symmetric $\left[\mathrm{SiO}_{4}\right]^{4-}$ tetrahedra possess no dipole moment (as the dipoles of the four $\mathrm{Si}-\mathrm{O}$ bonds cancel each other) and thus would not contribute to the overall dipole moment of a nanocluster. In our small nanoclusters, these silica tetrahedra only display small deviations from their ideal structure (Escatllar et al. 2019) and thus do not tend to make a significant contribution to the overall calculated dipole moments. We also note that, although the structures of silicate nanoclusters are non-crystalline, often we find some $\left[\mathrm{SiO}_{4}\right]^{4-}$ tetrahedra that are orientationally aligned (see atomistic structures in Fig. 3). Overall, the presence of large dipole moments in nanosilicates is clearly not simply due to random orientations of Si-O bonds as suggested by other authors (Hoang et al. 2016).

Inspecting the atomistic structures of our nanoclusters and their associated electrostatic potential we find that generally the regions with largest negative electrostatic potential correspond to surface $\mathrm{Mg}^{2+}$ cations positions, while areas of positive electrostatic potential are associated with surface oxygen anions locations. This tendency can be understood from the lower number of neighbours for ions at the nanocluster surface and a resultant lowering of the shielding of the electrostatic field for these ions in directions away from the nanocluster. Asymmetries in the positions of anions and cations near a nanocluster surface will thus tend to produce an overall nanocluster dipole. Conversely, the short-range dipoles due to alternating charges of cations and anions within the nanocluster will tend to be both counteracted by dipoles of neighbouring ion pairs and/or be shielded by the polarisation of the surrounding charge density. The energetic stability of a silicate nanocluster mainly depends on the number and strength of interactions due to ionic bonding. Although asymmetric arrangements of cations and anions tend to give rise to dipoles, generally in a small nanocluster the energetic cost to maintain an overall dipole is outweighed by the sum of bonding interaction energies. Overall, we find that only very few nanocluster isomers have symmetric atomistic structures which naturally lead to a zero overall dipole. In our set of lowest energy nanoclusters, the P-6 nanocluster is an example of a symmetric structure with no dipole. To highlight this is not a typical situation, in Fig. $4 \mathrm{~A}$ we plot the relative energetic stabilities against the dipole moments of 180 low energy $\left(\mathrm{MgSiO}_{3}\right)_{6}$ isomers, clearly showing the anomalously low dipole of the P-6 structure. Similarly, for olivine nanoclusters in Fig. 4B we show the analogous plot for 180 low energy $\left(\mathrm{Mg}_{2} \mathrm{SiO}_{4}\right)_{6}$ isomers where the lowest energy O-6 nanocluster has a finite dipole and where only two metastable isomers have dipole moments close to zero. In both cases, we note that there is no correlation between relative energetic stability and dipole moment, confirming that the energetic cost of maintaining a dipole in nanosilicates is small compared to the internal bonding energy.

While the lowest energy nanocluster structure would be the most populated in thermodynamic equilibrium, processing in astronomical environments could generate metastable isomers which can be kinetically trapped for long periods of time. As such, rather than the dipole moment of only the lowest energy nanocluster, a better overall estimate of the dipole possessed by astronomical nanosilicates may be to take an average dipole moment over a set of nanocluster isomers. In Figs. 4A and B the vertical red dashed lines indicate the median of the dipole moment all corresponding isomers. In both cases, the median dipole value is found to be larger than the dipole moment of the respective lowest energy nanocluster. Although this result is expected for the P-6 case for which the dipole moment is zero, the dipole moment the O-6 nanocluster is also significantly lower than the median value. These results indicate that our calculated dipole moments for the lowest energy nanocluster for each size is likely to be a lower bound estimate with respect to an average value taken with respect to many nanocluster isomers for that size. In Fig. 4C we compare the $\mu$ (Debye) values against number of atoms ( $N_{\text {atoms }}$ ) for all our O- $N, \mathrm{P}-N$ and $\mathrm{SiO}-N$ nanoclusters in their neutral state. The grey shaded area in the plot highlights the region for which nanosilicates would not significantly contribute to the AME (i.e. $\mu\left(N_{\text {atoms }}\right)<\beta \sqrt{N_{\text {atoms }}}$ for $\beta=0.3$ ), as estimated in previous studies (Hensley \& Draine 2017; Hoang et al. 2016). The black dashed lines indicate the previously estimated upper and lower limits for dipole moments (i.e., $\beta=1.0$ and $\beta=0.3$ ) for nanosilicates in the two previous references. These upper and lower limits are also indicated in Figs. 4A and B by vertical black dashed lines. Overall, the $\mu\left(N_{\text {atoms }}\right)$ values of the olivine and pyroxene nanoclusters show little regularity with respect to size. The $\mu\left(N_{\text {atoms }}\right)$ values of the smaller P- $N$ and O- $N$ nanoclusters have some tendency to fluctuate more at smaller sizes probably due to the greater proportion of surface to interior atoms in such species. The O-4 nanocluster has a particularly large dipole moment for its size, which is even larger than that of the O-1 nanocluster. As noted above, the origin of such large dipole moment seems to be the presence of a low coordinated surface $\mathrm{Mg}$ cation (see Fig. 3) which is not counteracted by any other ion. Generally, the majority of O- $N$ and P- $N$ nanoclusters (except for the symmetric P-2 and P-6 nanoclusters) possess $\mu$ values, 
A)

P-6 isomers

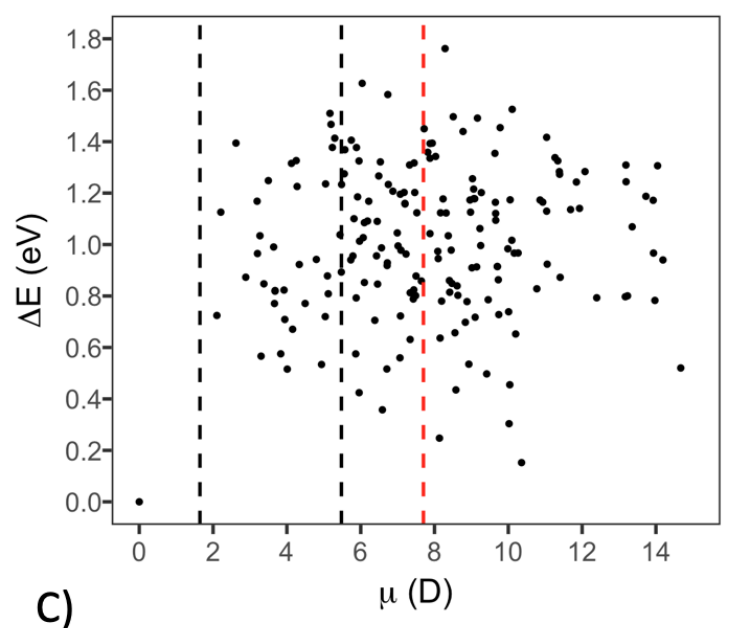

B) O-6 isomers

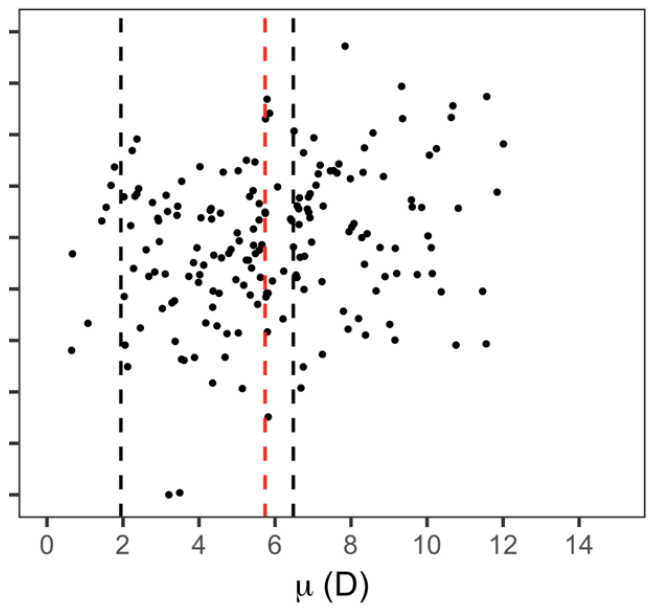

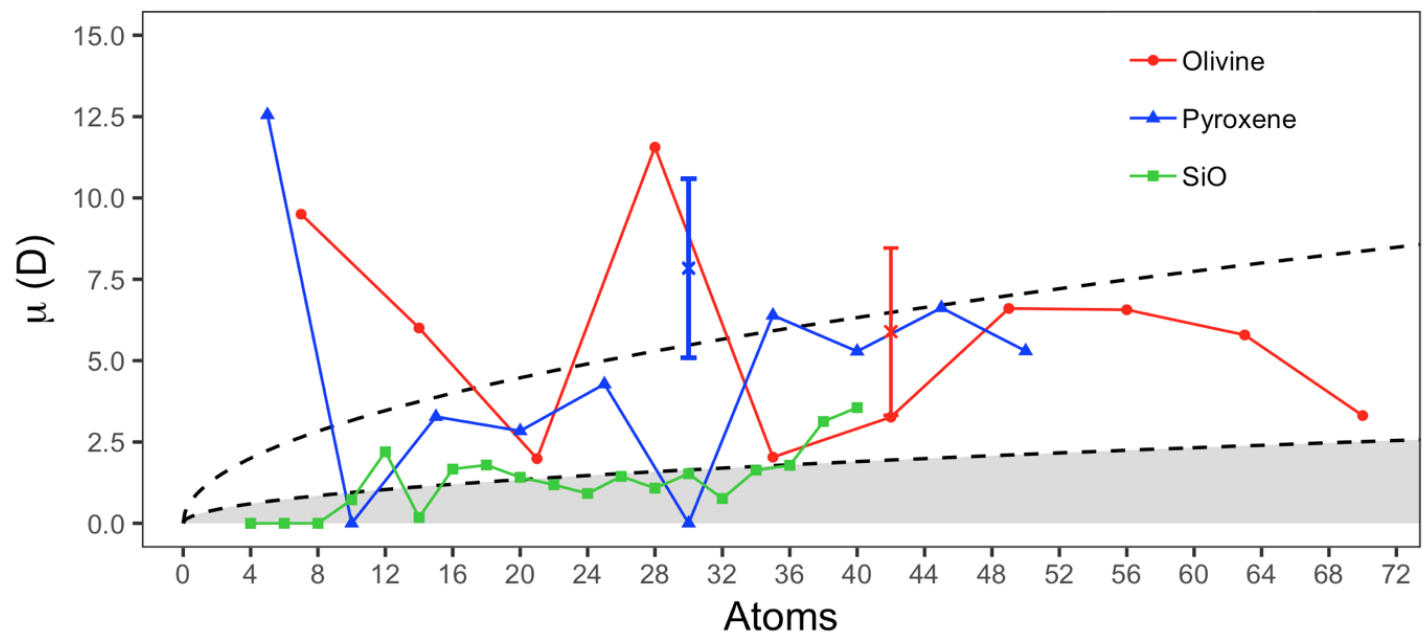

Fig. 4. Top: values of $\mu$ (Debye) against relative energy (eV) of nanocluster isomer with respect to the lowest energy structure for P-6 isomers $(A)$ and O-6 isomers $(B)$. In each plot, the black dashed lines indicate the lowest and highest previously estimated dipole moments for nanosilicates (Hoang et al. 2016), while the red dashed lines mark the median value calculated for each data set. (C) Dipole moments $\mu$ for O- $N$ (red circles), P- $N$ (blue triangles) and SiO- $N$ (green squares) nanoclusters with respect to the number of atoms $N_{\text {atoms }}$. The blue and red crosses correspond to the average dipole $\mu$ values for the P-6 and O-6 isomer shown in $(A)$ and $(B)$ with the error bars indicating the variance in $\mu$ values for each set. The grey shaded area corresponds to the region where the dipole moment is too small for nanosilicates to contribute substantially to the AME. The two dashed lines correspond to the limits in $(A)$ and $(B)$.

which, although varying in a rather irregular manner, are above the lower value deemed significant for contributing to the AME (i.e. $\beta=0.3$ ). Many of these nanoclusters have dipole moments that are close to, and sometimes above, the upper estimated limit (i.e. for $\beta=1.0$ ) for nanosilicates. As noted above, as our $\mu$ values are calculated for the lowest energy nanoclusters, this means that they are likely lower estimates of median dipole moments for each size and composition. In Fig. 4C we also include median and variance of the set of $\mu$ values for $\left(\mathrm{MgSiO}_{3}\right)_{6}\left(N_{\text {atoms }}=\right.$ 30) and $\left(\mathrm{Mg}_{2} \mathrm{SiO}_{4}\right)_{6}\left(N_{\text {atoms }}=42\right)$ isomers plotted in Figs. $4 \mathrm{~A}$ and $\mathrm{B}$. In both cases, the dipole moment of the original lowest energy nanocluster would be shifted to near or above the notational upper limit line. Clearly, however, whether we take median $\mu$ values or not, our Mg-rich olivine and pyroxene nanoclusters have dipoles that are sufficiently large to significantly contribute to the AME.

In contrast to the O- $N$ and $\mathrm{P}-N$ species, the neutral $\mathrm{SiO}-N$ nanoclusters generally display significantly lower dipole moments making them less likely to contribute to the AME. For many of the $\mathrm{SiO}-N$ nanoclusters the $\mu$ values are on, or below, the lower limit for AME significance. Only five of the nineteen $\mathrm{SiO}-N$ nanoclusters considered have sufficiently large dipole moments to be above this limit. The zero dipole of the first few $\mathrm{SiO}-N$ nanoclusters can be ascribed to their symmetric structures. However, for larger sizes $(\mathrm{SiO})_{N}$ nanoclusters tend to structurally segregate into oxygen-rich (i.e. $\mathrm{SiO}_{2}$-like) and silicon-rich regions in a side-by-side fashion (Bromley et al. 2016). Although, such structures are suitably asymmetric for possessing a large dipole, in the neutral state charge does not tend to localise on either segregated region. As such the only ionic charge imbalance come from the formation of $\mathrm{Si}-\mathrm{O}$ bonds in the oxygen-rich regions which, in the absence of network disrupting $\mathrm{Mg}^{2+}$ ions, tend to form symmetric $(\mathrm{SiO})_{x}$ rings and $\left(\mathrm{SiO}_{4}\right)^{4-}$ tetrahedra, both of which possess very low dipole moments.

As noted above, ISM silicates are likely to contain a small fraction of $\mathrm{Fe}^{2+}$ in their chemical structure, which has been 


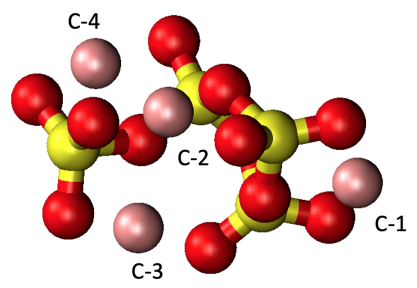

Fig. 5. Structure of the P-4 nanocluster with the different cation positions labelled.

Table 1. Dipole moment variation of the P-4 structure by substituting one $\mathrm{Mg}^{2+}$ by one $\mathrm{Fe}^{2+}$ in each of the four different sites (C-1 to C-4).

\begin{tabular}{ccc}
\hline \hline Structure & $\mu(\mathrm{D})$ & $\Delta \mu(\mathrm{D})$ \\
\hline Original P-4 & 2.83 & 0.00 \\
Fe C-1 & 3.55 & +0.72 \\
Fe C-2 & 1.56 & -1.27 \\
Fe C-3 & 3.06 & +0.23 \\
Fe C-4 & 2.34 & -0.49 \\
Fe mean & 2.63 & -0.2 \\
\hline
\end{tabular}

assumed to explain the polarization of starlight and polarized emission from aligned dust grains (Hoang et al. 2010). Although the present work does not deal with polarisation, the inclusion of $\mathrm{Fe}^{2+}$ could nevertheless affect the calculated dipole moments of the nanoclusters. As a test case we evaluate the change in the dipole moment induced by substituting one $\mathrm{Mg}^{2+}$ cation by a $\mathrm{Fe}^{2+}$ cation in the P-4 nanocluster in all four symmetrically inequivalent positions, $\mathrm{C}-1$ to $\mathrm{C}-4$ (see Fig. 5). We note that the energetic stability of $\mathrm{Fe}^{2+}$ substitution in each of the positions is very similar (difference of $<0.2 \mathrm{eV}$ ) and thus all could be occupied in a population of $\mathrm{Fe}^{2+}$-substituted P-4 nanoclusters. In Table 1 we show the dipole moments of the P-4 nanocluster with each $\mathrm{C} \mathrm{Mg}^{2+}$ cation substituted by a $\mathrm{Fe}^{2+}$ cation. The results show that the dipole moment of the $\mathrm{Fe}$ substituted small P-4 nanocluster can both increase and decrease with respect to the $\mathrm{Mg}$-pure original P-4 structure. In this case, we find a maximum variation of -1.27 Debyes. Although in this extreme case the dipole reduction is significant, the total dipole moment is still 1.56 Debyes and thus the nanocluster would still be a viable carrier of the AME. Overall, in this test case, the range and average change of dipole moment due to Fe-substitution would not be significant compared to the dipole variation expected due to structural isomerisation (see Figs. 4A and $\mathrm{B}$ ).

Charging of nanoclusters will also affect their electronic structure and hence their corresponding dipole moment. In addition, an excess charge can significantly affect the interaction between a nanocluster and its environment, influencing, for instance, the collisional cross-section or the grain temperature. In Draine \& Lazarian (1998), the effect of grain charging on the dipole is modelled by assuming that the extra charge is localized over a region of the grain with the charge centroid displaced from the centre of mass by $1 \%$ of the grain radius (see also Purcell 1975). Such a localized charge would then add to the intrinsic dipole moment by $\Delta \mu=\epsilon a_{x} \mathrm{Ze}$, with $\epsilon=0.01$ being the displacement of the charge centroid with respect to the centre of mass, $\mathrm{a}_{x}$ the grain radius and Ze the grain charge. The overall predicted effect is that charging a grain increases its dipole moment by a small amount. In Fig. 6 we report the difference in dipole
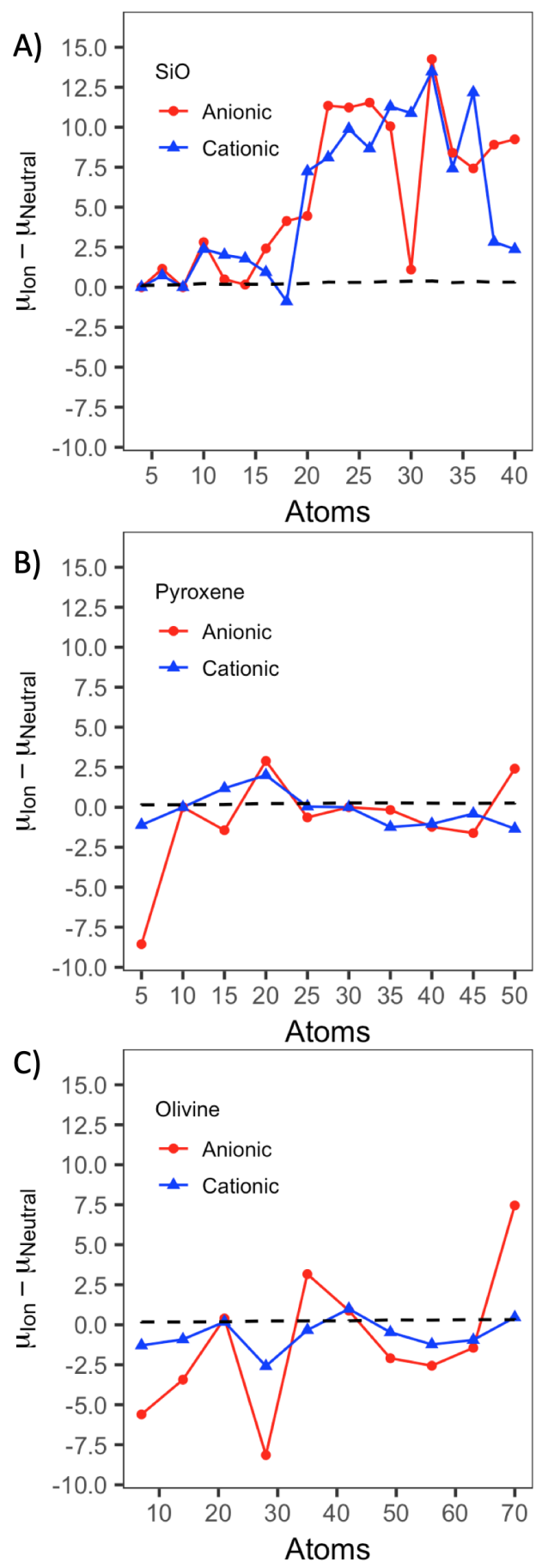

Fig. 6. Dipole moment (Debye) difference between ionic (red circles: anionic, blue triangles: cationic) and neutral states for the $\mathrm{SiO}$, pyroxene and olivine nanoclusters. The dashed line correspond to the increase of dipole moment using the model of Draine \& Lazarian (1998) and using the largest nanocluster axis to project the displacement.

moment in the \pm 1 anionic/cationic states of our nanoclusters with respect to that of corresponding neutral nanoclusters where the dashed lines indicate the increase in dipole predicted by classical top-down modelling (Draine \& Lazarian 1998). Clearly, in nearly all cases the changes in dipole we observe in our quantum chemical calculations are significantly larger and less regular in magnitude with respect to size and composition than the classical estimates. Typical changes in the dipole moment for the classical model are of the order of $0.2 \mathrm{D}$. Thus, due to the large 
A)

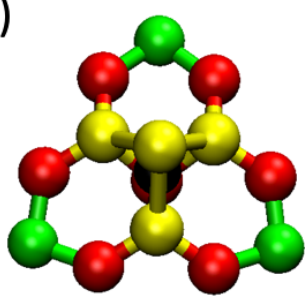

B)

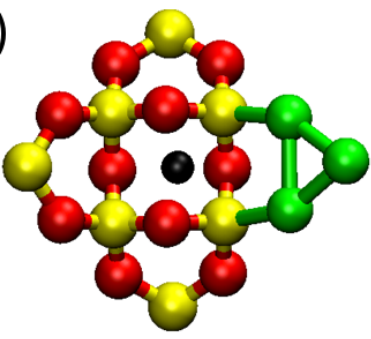

Fig. 7. Chemical structures of anionic nanoclusters for: $(A)(\mathrm{SiO})_{7}$ and (B) $(\mathrm{SiO})_{10}$ nanoclusters. The black dots correspond to the centres of mass. Oxygens are shown as red, while silicon is shown as yellow or green. The green colour indicates that the $\mathrm{Si}$ centre in question charge possesses a larger than average negative charge.

range in the difference of dipole moments in Fig. 6 the prediction of classical model appears as a horizontal line close to zero. Even though it seems intuitive that adding a charge to a nanocluster would increase its dipole moment, the results of our accurate electronic structure calculations show that this is not necessarily the case. In fact, for most of the O- $N$ and P- $N$ nanoclusters a change in the charge state results in a diminished dipole moment, suggesting that the added charge allocates itself to counteract the existing dipole of the neutral nanocluster. This is most clearly exhibited for the O-4 nanocluster which, as a neutral nanocluster, has a large dipole moment. However, once the O-4 nanocluster becomes charged most of the dipole moment is counteracted. Conversely, charged $\mathrm{SiO}-N$ nanoclusters with $N \geq 10$ exhibit large and consistent increases in their dipole moments. The reason for such behaviour in these nanoclusters is their asymmetric segregated nature. Although in the neutral charge-balanced state these segregated nanoclusters have low dipole moments (see above) excess charge localizes on either the O-rich or Si-rich side of the nanocluster giving rise to a spatially asymmetric charge distribution and the generation of a significant dipole moment. We note that for $N<10$ the SiO- $N$ nanoclusters have a more symmetric structure and the additional charge is distributed in a more balanced way, thus not leading to large dipole increases. In Fig. 7 we show structures of the $(\mathrm{SiO})_{7}$ and $(\mathrm{SiO})_{10}$ nanoclusters, where we colour in green the atoms that receive most of the electron density from an added electron. The $(\mathrm{SiO})_{7}$ cluster distributes the electron density on three peripheral $\mathrm{Si}$ atoms in a symmetric fashion that leads to little change in the overall dipole moment. However, in the $(\mathrm{SiO})_{10}$ nanocluster the added electron is completely localized on the Si-rich region which generates a large difference between the centre of mass and centre of charge, dramatically increasing the dipole moment.

\section{Ionization potentials and electron affinities}

From our quantum chemical calculations we can also extract the electron affinity (EA) and the ionization potential (IP) of our nanoclusters. These two parameters can be used to help understand the charge state of the nanoclusters in various astrophysical environments as well as to determine the temperature of the ISM (Weingartner \& Draine 2001a). Figure 8 provides the IP and EA values of the $\mathrm{O}-N, \mathrm{P}-N$ and $\mathrm{SiO}-N$ nanoclusters versus their radius in $\AA$. Here we calculate the radius using the average of the moments of inertia (i.e. modelling the nanocluster as a sphere with homogeneous density). Although our nanoclusters are not perfectly spherical (see above) we convert them to a spherical

shape in order to better compare our IP and EA values with values calculated using the classical electrostatic based equations reported in Weingartner \& Draine (2001a). For the IP values, the discrepancies between our quantum chemical calculations and classical electrostatic estimates are largest for both the smallest $\mathrm{O}-1$ and $\mathrm{P}-1$ nanoclusters $(>3.5 \mathrm{eV})$. With increasing nanocluster size our calculated IP values for the O- $N$ and $\mathrm{P}-N$ series gradually decrease linearly with respect to the nanocluster radius whereas the classical electrostatic values decrease proportionally to the inverse nanocluster radius. For the P- $N$ set of nanoclusters, our calculated values meet the classical estimated tendency at a radius of $\sim 4.0 \AA$ and match well up to a radius of $\sim 4.7 \AA$. For the $\mathrm{O}-N$ nanoclusters, the IP values as predicted by our quantum chemical calculations tend to be approximately $0.5 \mathrm{eV}$ lower than those of the P- $N$ nanoclusters. As such, the IP values for the $\mathrm{O}-N$ nanoclusters would likely match the classical prediction at nanocluster size larger than of those considered in this study (for the P-10 nanocluster the difference is $\sim 0.5 \mathrm{eV}$ ). For the $\mathrm{SiO}-N$ nanoclusters our calculated IP values are always significantly below the classical approximation, where, even for the largest seven $\mathrm{SiO}-N$ nanoclusters, the difference is $>3 \mathrm{eV}$.

The quantum chemically calculated EA values for both O- $N$ and $\mathrm{P}-N$ nanoclusters appear to follow a similar gradually decreasing tendency with respect to increasing radius, with both sets of values seeming to reach a plateau at $0.86 \mathrm{eV}$ for the largest sizes. Conversely, the classical electrostatic values follow a negative inverse radius relationship with respect to nanocluster radius which crosses the limiting plateau EA value over a radius range of $\sim 3-5 \AA$. Moving above and below this nanocluster size range the discrepancy between the classical electrostatic predicted EA values and the quantum chemical values increases. For the $\mathrm{SiO}-N$ nanoclusters the general tendency is for the EA values to increase in a fairly consistent fashion with respect to the classical approximation. Generally, the magnitudes of the calculated $\mathrm{EA}$ values for smaller $\mathrm{SiO}-N$ nanoclusters tend to be higher than those predicted by the classical estimates. However, for the larger $\mathrm{SiO}-N$ nanoclusters, for $N=10-19$, the calculated EA values oscillate above and below the classical prediction.

The IP and EA values reported by Weingartner \& Draine (2001a) are used for silicate nanocluster sizes with a $>3.5 \AA$ (see solid portion of black lines in Fig. 8). Comparing our calculated IP values with those of Weingartner \& Draine (2001a) in this region for the $\mathrm{O}-N$ and $\mathrm{P}-N$ nanoclusters we obtain maximal differences of $\sim 1 \mathrm{eV}$ for nanoclusters of $3.5 \AA$ radius and $\sim 0.5 \mathrm{eV}$ for a $5 \AA$ radius. However, for the $\mathrm{SiO}-N$ nanoclusters the respective energetic differences are $\sim 6$ and $\sim 4 \mathrm{eV}$. For the EA values, the corresponding energy differences for the $\mathrm{O}-N$ and $\mathrm{P}-N$ nanoclusters are $<0.5 \mathrm{eV}$ for nanoclusters of $3.5 \AA$ radius, and $\sim 0.8 \mathrm{eV}$ for $5 \AA$ radius nanocluster. For the $\mathrm{SiO}-N$ nanoclusters the respective energy differences are $\sim 3$ and $\sim 0.5 \mathrm{eV}$.

In general, the size dependence of the values of the IP and EA as predicted by classical electrostatic arguments is not in very good overall agreement with that of more accurate quantum chemical calculations. However, for $\mathrm{O}-N$ and $\mathrm{P}-N$ silicate nanoclusters with a radius close to $\sim 4 \AA$ the match between the EA and IP values from the two types of calculations is quite reasonable. For the IP values this match is fairly well maintained for larger nanocluster sizes, whereas for the EA values the correspondence deteriorates. For the $\mathrm{SiO}-N$ nanoclusters our quantum chemically calculated IP values are always significantly below those predicted by the classical approximation although for larger sizes the EA values predicted by both methods tend to be in closer agreement. 
A)

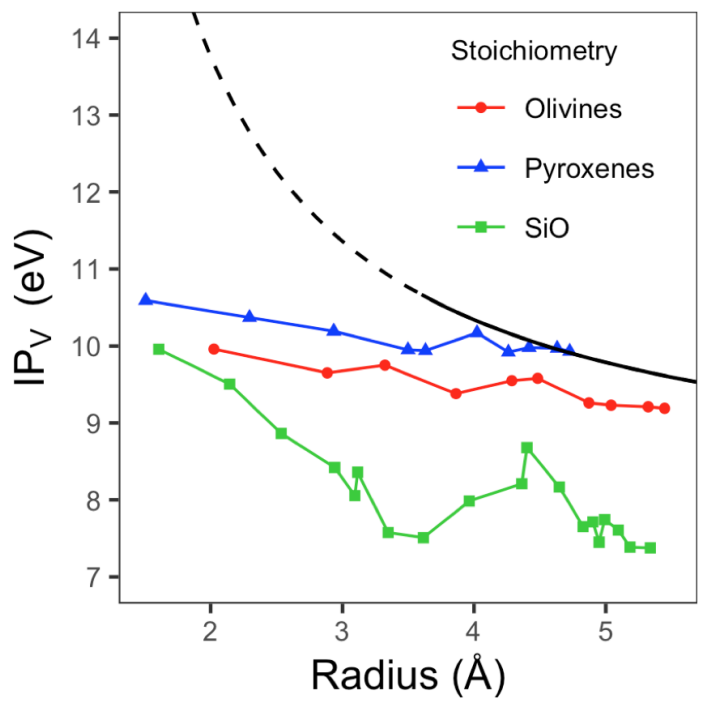

B)

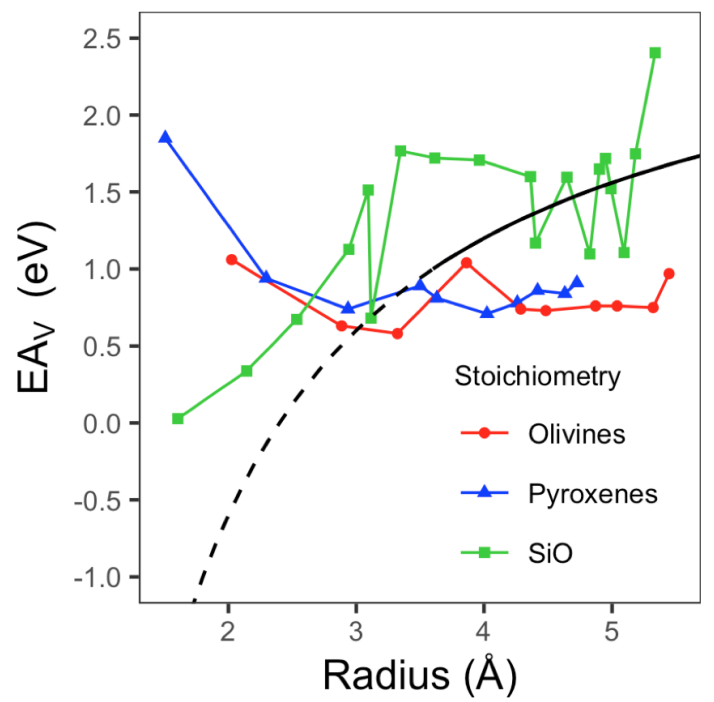

Fig. 8. $\mathrm{IP}_{V}(A)$ and $\mathrm{EA}_{V}(B)$ for the O- $N$ (red circles), $\mathrm{P}-N$ (blue triangles) and $\mathrm{SiO}-N$ (green squares) nanoclusters. The dashed and solid black line corresponds to the classically derived equations used in Weingartner \& Draine (2001a). Note that only the solid part of the black line corresponds to values applied to nanosized grains in Weingartner \& Draine (2001a).

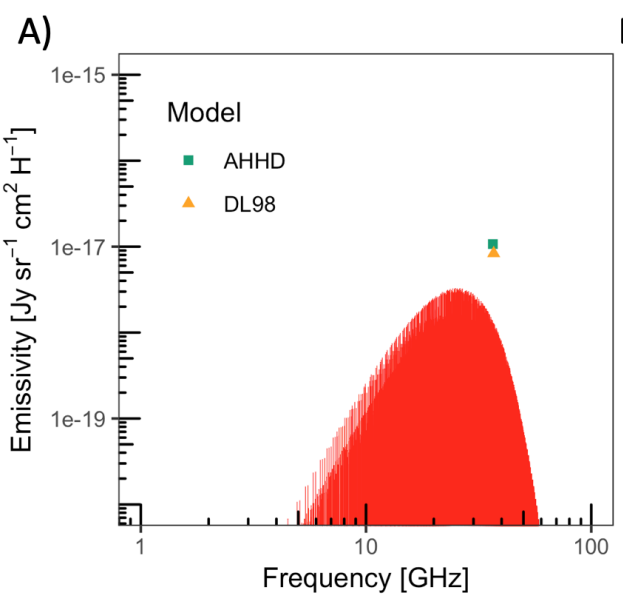

B)

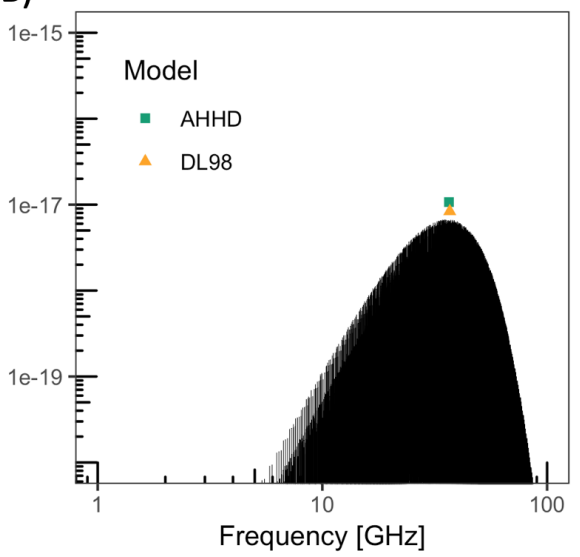

C)

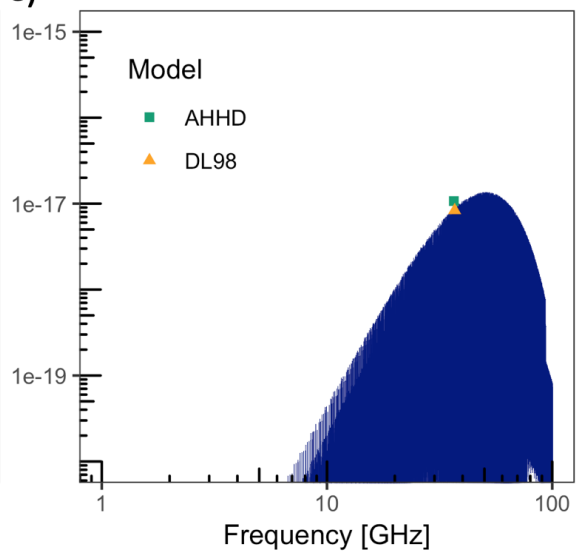

Fig. 9. Emissivity of a representative grain of $\left(\mathrm{Mg}_{2} \mathrm{SiO}_{4}\right)_{3}$ containing $1 \%$ of the total Si budget at rotational temperatures of $T=10$ (red, left), 20 (black, middle) and 40 (dark blue, right) $\mathrm{K}$ under MC conditions as described in Draine \& Lazarian (1998). The maximum emissivity of the models of Ali-Haïmoud et al. (2009, AHHD) and Draine \& Lazarian (1998, DL98) are also plotted.

\section{Microwave emission}

The quantum mechanical derived data in this work allows us to derive the microwave emission of our nanosilicates. Figure 9 shows the predicted emissivity of a population of representative O-3 nanoclusters under general assumed conditions of MCs as described in Draine \& Lazarian (1998), using three rotational temperatures of $T_{\text {rot }}=T_{\text {dust }}=10,20$ and $40 \mathrm{~K}$ and containing a fraction of $1 \%$ of total Si. Thus, the only difference in the three models corresponds to changes in temperature. As expected, an increase in temperature causes an increase of emissivity and peak frequency. For the temperature of $20 \mathrm{~K}$, both peak position and emissivity are in very good agreement with the models of Draine \& Lazarian (1998, model DL98) and Ali-Haïmoud et al. (2009, model AHHD). Again, we highlight that both cited methodologies compute the rotational distribution of the grains by estimating the influence of numerous physical processes involved in accelerating and decelerating grain rotation. Thus, such models can be applied to a wide range of environments where rotation may be above or below the thermal rotation rate. Several parameters are required for such models, both for the environment and the grain. Nevertheless, in some circumstances, the rotational energy distribution will still follow the Boltzmann distribution. In Appendix A we show that, under general MC conditions, the present study under the assumption of LTE slightly overestimates the emission in comparison with the detailed models of DL98 and AHHD by $\sim 40 \%$. On the other hand, the O-3 nanocluster possesses one of the lowest non-zero dipoles moments in our considered set of nanocluster isomers. In Fig. 4 we show that the average dipole moments of a population of P-6 and O-6 nanocluster isomers is larger than that of the lowest energy P-6 and O-6 nanocluster isomer. Assuming this is a general tendency, we should then use a larger dipole moment value for a representative average O-3 nanocluster. Doing so would cause the calculated emission to be larger than shown in Fig. 9 and would imply that a lower 
A)

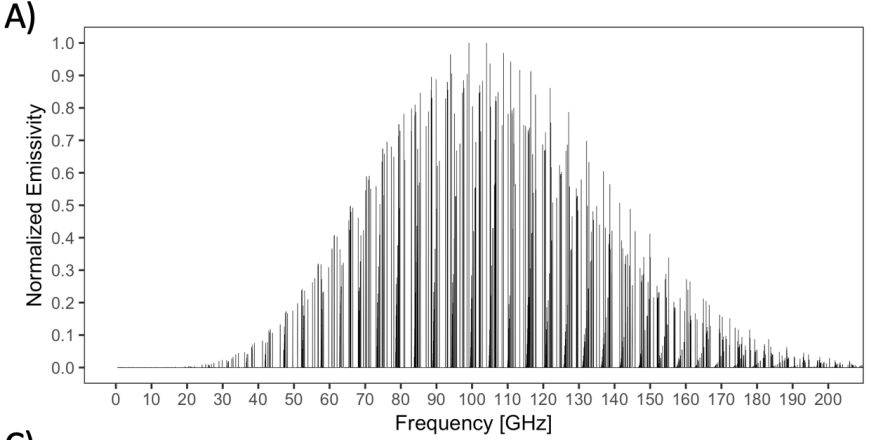

C)

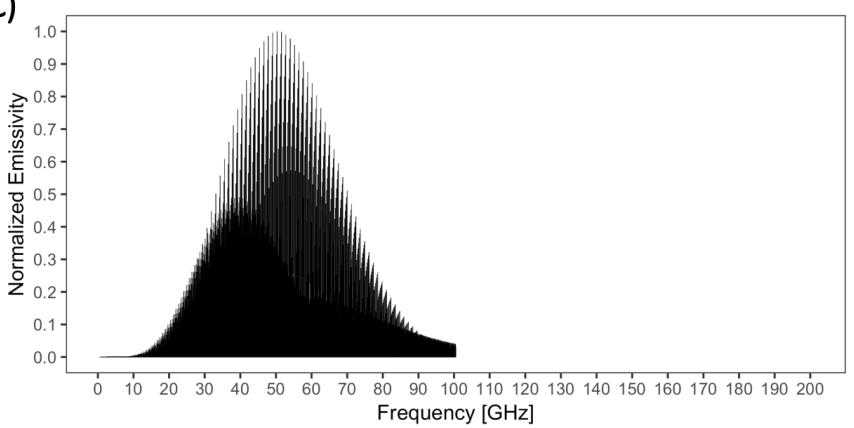

B)

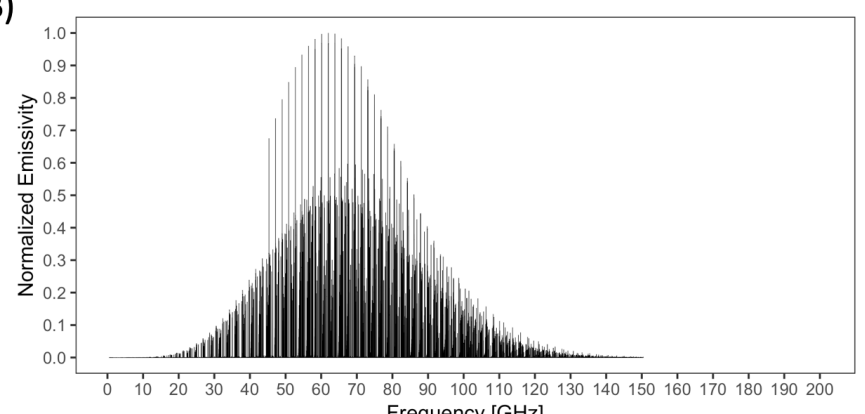

D)

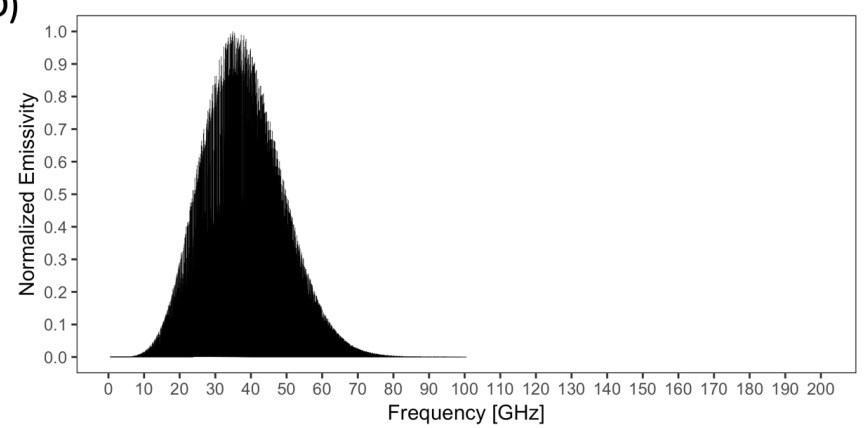

Fig. 10. Calculated microwave emission spectra of the P-1 $(A), \mathrm{O}-1(B)$, O-2 $(C)$ and O-3 $(D)$ nanoclusters at $T=20 \mathrm{~K}$ normalized so that the most intense peak has unit intensity. For P-1 no clear separation between lines is found. For O-1, the most intense lines are separated by a line spacing of $1.85 \mathrm{GHz}$, which lowers to $1.3 \mathrm{Ghz}$ for O-2. For O-3 the density of states becomes too large to identify a line-spacing, although some individual lines appear.

abundance of nanoclusters would be required to produce this emission.

Li \& Draine (2001) argued that silicate nanoclusters with diameters $\leq 1.0 \mathrm{~nm}$ radii could not contain more than $15 \%$ of $\mathrm{Si}$ in the ISM based on the lack of IR signatures attributed to silicates. Based on this constraint, Hensley \& Draine (2017) and Hoang et al. (2016) argued that the entirety of the AME could be attributed to such silicate nanoclusters as long as they possess sufficiently large dipole moments. We note that such constraints should be viewed with caution as the IR spectra of silicate nanoclusters can not generally be extrapolated from those of bulk silicates (Zamirri et al. 2019; Escatllar et al. 2019). Herein, having access to the silicate nanocluster dipole moment, we can, instead, find the appropriate population of nanoclusters that would be required to account for the observed AME. Our modelling requires only $1 \%$ of the total Si budget to reproduce AME predictions of other models in MCs. In this way, we have an alternative bottom-up constraint on silicate nanocluster abundances based on microwave observation.

As noted in Draine \& Lazarian (1998), the use of classical mechanics to describe the microwave emission can be justified if a grain is above a certain size or temperature. However, several of our grains are small enough to be effectively considered molecules, such as P-1, O-1 and a fraction of the smaller SiO$N$ species. In addition, the high resolution achievable by radio telescopes (e.g. $0.4 \mathrm{MHz}$, Ali-Haïmoud et al. 2015) requires us to evaluate the possibility that, even if the overall spectral shape of the emission can be described by classical mechanics, at such high resolutions some hint of structure could also be found. Although a full analysis of the microwave spectra of the nanoclusters is beyond the scope of the present work, in Fig. 10 we show the calculated normalized (i.e, strengths are relative to the most intense signal) microwave spectra for the small P-1, O-1, O-2 and O-3 nanoclusters at MC conditions
( $20 \mathrm{~K}$ rotational temperature). P-2 was not considered as its high symmetry leads to a zero dipole moment, and thus no emission. We find that the P-1 spectrum shows a complicated pattern of well-separated peaks (see Fig. 10A) with no clear constant line-separation. The O-1 nanocluster also shows molecular-like emission spectrum, but in this case the main peaks are much more consistent showing a spacing of $\sim 1.8 \mathrm{GHz}$. With increasing nanocluster size, the density of states increases and the peak separation becomes smaller and the spectra become an almost continuous (see Fig. 10D).

In Fig. 11, we compare the microwave emission spectra of Mg-pure olivine and pyroxene nanoclusters with three sizes (small: one monomeric unit of olivine and pyroxene, medium: P-4 and O-3 nanoclusters containing 20 and 21 atoms respectively, and large: P-10 and O-10 nanoclusters, containing 50 and 70 atoms respectively) in conditions representative of MCs $(T=$ $20 \mathrm{~K}$ ). The fraction of the total Si budget in the nanoclusters is taken to be $1 \%$ for the medium and large nanoclusters and $10^{-5}$ for the smallest ones. With increasing size we find that the emissivity rapidly decreases. The plots in Fig. 11 show that, as expected, an increase in mass is correlated with a decrease in the magnitude of the rotational constants (see Table B.1), and thus a smaller spacing of the rotational levels. Hence, the spectral peaks are displaced towards lower frequencies with an increasing number of atoms. It is clear from Fig. 11 that the microwave emission from all the nanoclusters in the size range we consider overlaps with the observed frequency range of the AME at $T=20 \mathrm{~K}$. The smallest nanoclusters have emission covering most of the observable AME frequency range but tend to peak at higher frequencies than those observed. At $20 \mathrm{~K}$, we find that the emissivity of the smallest P-1 and O-1 nanoclusters peaks at a frequency close to $100 \mathrm{GHz}$. As AME observations generally have a maximum emissivity in the range between 20 and $30 \mathrm{GHz}$, it follows that the population fraction of the $\mathrm{O}-1$ and $\mathrm{P}-1$ nanoclusters must 

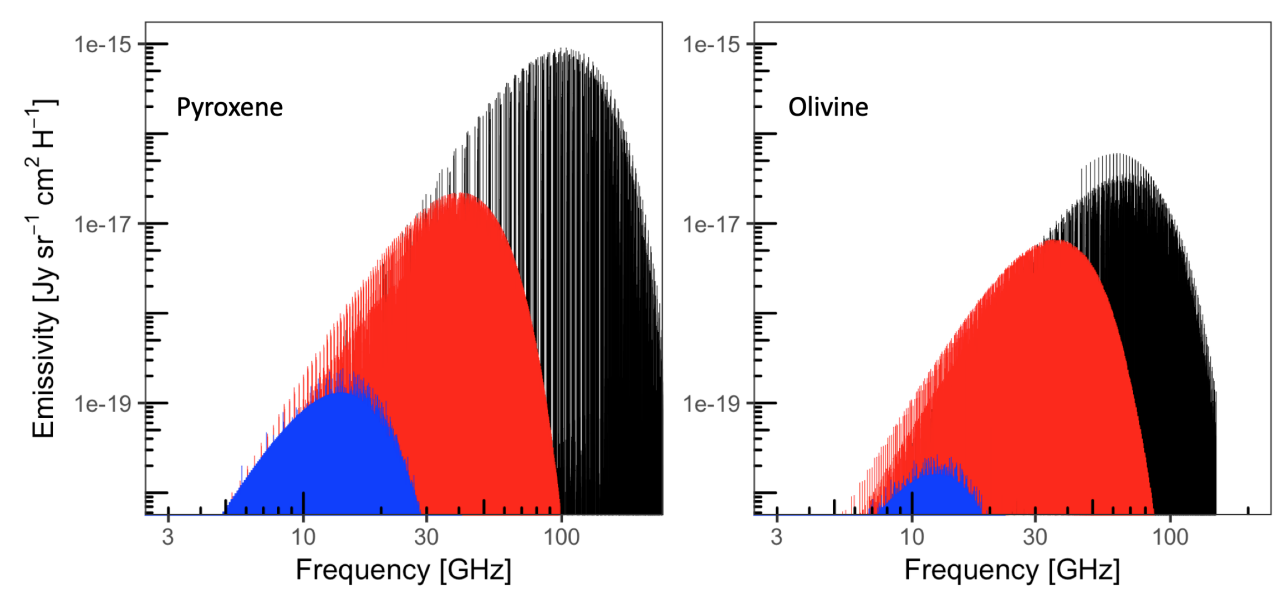

Fig. 11. Calculated microwave emission spectra for the O- $N$ and P- $N$ for small (i.e. $N=1$ for O- $N$ and P- $N$, black), large (i.e. $N=10$ for O- $N$ and $\mathrm{P}-N$, blue) and medium-sized nanocluster containing $\sim 20$ atoms ( $N=3$ for O- $N, N=4$ for P- $N$ and $N=10$ for SiO- $N$, red) under MC ( $T=20 \mathrm{~K})$ conditions. The emissivity is calculated using a fraction of 0.01 of $\mathrm{Si}$ in the medium and large nanoclusters and 1e-5 for the smallest nanoclusters.

be smaller than the $10^{-5}$ used in our modelling. Conversely, from Figs. 9 and 11 it can be seen that Mg-rich silicate nanoclusters containing 20 to 30 atoms can best account for the observed AME spectral shape in MC conditions. Since larger rotational temperatures imply emission at higher frequencies, larger nanoclusters ( $~ 50$ to 70 atoms) could also then be significant contributors to the AME. With respect to observation, the monomeric species P-1 and O-1 would appear as the most easily detectable species due to the large separation between their spectral peaks and their large emissivities.

\section{Conclusions}

While the evidence that small spinning dust grains are the source of the AME seems to be well established, the nature of the potential nanosized carriers is still under discussion. In this work we perform accurate bottom-up quantum chemical calculations of the properties of astronomically relevant silicate clusters of $\mathrm{Mg}_{2} \mathrm{SiO}_{4}$ (olivine), $\mathrm{MgSiO}_{3}$ (pyroxene) and $\mathrm{SiO}$ (silicon monoxide) with diameters $\leq 1 \mathrm{~nm}$, with the main aim of clarifying their importance as carriers of the AME. Our results provide strong evidence that silicate nanoclusters, if present, would be a major component of the AME. Specifically, our results show that:

1. Mg-rich olivine and pyroxene silicate nanoclusters are mainly prolate shaped, with no clear tendency to become spherical with increasing size.

2. The size dependency of IP and EA values calculated by classical electrostatic estimates are generally not in good agreement with those from quantum chemical calculations. However, for olivine and pyroxene nanoclusters with radii close to $4 \AA$ the agreement between both approaches is reasonable.

3. The dipole moments of neutral $\mathrm{Mg}$-rich silicate clusters of both olivine $\left(\mathrm{Mg}_{2} \mathrm{SiO}_{4}\right)$ and pyroxene $\left(\mathrm{MgSiO}_{3}\right)$ compositions are large and well above the estimated minimum for silicates to contribute to the AME. Our statistical analysis of dipole moments for over 180 nanocluster isomer structures for two sizes, $\left(\mathrm{Mg}_{2} \mathrm{SiO}_{4}\right)_{6}$ and $\left(\mathrm{MgSiO}_{3}\right)_{6}$, shows less than $4 \%$ have a dipole moment below the required values. $\mathrm{SiO}$ clusters on the other hand tend to have much lower dipole moments and are less likely to be significant contributors to the AME.
4. Changing the charge state of the nanoclusters can significantly increase or decrease the dipole moment of a neutral nanocluster. For O- $N$ and P- $N$ nanoclusters and the smallest SiO- $N$ particles (with $N<10$ ) ionisation generally lowers the dipole moment of the particle, although the resulting dipoles are still large enough to significantly contribute to the AME. For the segregated $\mathrm{SiO}-N$ particles $(N>10)$ the charge is generally localized in the Si-rich part of the nanocluster, generating a large separation between the centre of mass and centre of charge, thus increasing the dipole moment. In such circumstances, charged $\mathrm{SiO}-N$ nanoclusters have larger dipole moments than similar sized magnesium silicate nanoclusters.

5. Substituting a small fraction of $\mathrm{Mg}^{2+}$ cations by $\mathrm{Fe}^{2+}$ cations can both increase or decrease the dipole moment of a magnesium silicate nanocluster. Although this effect can be fairly large for individual substitutions, it appears that the overall effect does not tend to significantly diminish the magnitude of nanocluster dipole moments. In particular, consideration of $\mathrm{Fe}^{2+}$ is very unlikely to affect our overall confirmation that silicate nanoclusters have sufficiently large dipole moments to be carriers of the AME.

6. Using a Boltzmann distribution to describe the population of the rotational levels we find that we only require $1 \%$ of the total $\mathrm{Si}$ abundance to be contained in Mg-rich silicate nanoclusters in order to reproduce the results of previously published AME models. Thus, we find that the abundance of silicate nanoclusters is better constrained by microwave spectra than IR spectra.

7. By virtue of their detailed and, in principle, resolvable microwave emission spectra, smaller silicate nanoclusters (e.g. P-1, O-1, O-2 and any isomer having <20 atoms) are primary candidates that could provide direct observable evidence of silicate nanoclusters in different ISM phases as well as to allow determine dust temperatures.

Acknowledgements. We acknowledge financial support from the Spanish MINECO/FEDER CTQ2015-64618-R and MICIUN RTI2018-095460-B-100 research projects, the Ministerio de Ciencia, Innnovación y Universidades (MCIU) Spanish Structures of Excellence María de Maeztu program through grant MDM-2017-0767 and the Generalitat de Catalunya (grants 2017SGR13 and XRQTC). The Red Espanola de Supercomputación (RES) is also acknowledged for the provision of supercomputing time. We also acknowledge the EU COST Action (CM1401) - Our Astro-Chemical History. We also thank an anonymous referee and Prof. Miguel Gonzalez for their helpful and constructive comments. 


\section{References}

Adamo, C., \& Barone, V. 1999, J. Chem. Phys., 110, 6158

Ade, P. A. R., Aghanim, N., Arnaud, M., et al. 2011, A\&A, 536, A20

Agladze, N. I., Sievers, A. J., Jones, S. A., Burlitch, J. M., \& Beckwith, S. V. W. 1996, ApJ, 462, 1026

Ali-Haïmoud, Y. 2013, Adv. Astron., 2013, 1

Ali-Haïmoud, Y., Hirata, C. M., \& Dickinson, C. 2009, MNRAS, 395, 1055

Ali-Haïmoud, Y., Pérez, L. M., Maddalena, R. J., \& Roshi, D. A. 2015, MNRAS, 447,315

Barone, V. 2005, J. Chem. Phys., 122, 014108

Blum, V., Gehrke, R., Hanke, F., et al. 2009, Comput. Phys. Commun., 180, 2175

Bromley, S. T., Gómez Martín, J. C., \& Plane, J. M. C. 2016, Phys. Chem. Chem. Phys., 18, 26913

Casassus, S., Cabrera, G. F., Forster, F., et al. 2006, ApJ, 639, 951

Catlow, C. R. A., Bromley, S. T., Hamad, S., et al. 2010, Phys. Chem. Chem. Phys., 12, 786

Das, H. K., Voshchinnikov, N. V., \& Il'in, V. B. 2010, MNRAS, 404, 265

Dickinson, C., Ali-Haïmoud, Y., Barr, A., et al. 2018, New Astron. Rev., 80, 1

Draine, B. T., \& Fraisse, A. A. 2009, ApJ, 696, 1

Draine, B. T., \& Hensley, B. S. 2016, ApJ, 831, 59

Draine, B. T., \& Lazarian, A. 1998, ApJ, 508, 157

Draine, B. T., \& Lazarian, A. 1999, ApJ, 512, 740

Erickson, W. C. 1957, ApJ, 126, 480

Escatllar, A. M., Lazaukas, T., Woodley, S. M., \& Bromley, S. T. 2019, ACS Earth Space Chem., 3, 2390

Finkbeiner, D. P., Schlegel, D. J., Frank, C., \& Heiles, C. 2002, ApJ, 566, 898

Fogerty, S., Forrest, W., Watson, D. M., Sargent, B. A., \& Koch, I. 2016, ApJ, 830,11

Frisch, M., Trucks, G., Schlegel, H., et al. 2016, Gaussian 16 Revision C.01 (Wallingford CT, Gaussian Inc.)

Gobrecht, D., Cherchneff, I., Sarangi, A., Plane, J. M. C., \& Bromley, S. T. 2016, A\&A, 585, A6

Gordy, W., \& Cook, R. L. 1984, Microwave Molecular Spectra, 3rd edn. (Hoboken: Wiley-Interscience Publication), 747

Goumans, T. P. M., \& Bromley, S. T. 2012, MNRAS, 420, 3344

Greaves, J., Scaife, A., Frayer, D., et al. 2018, Nat. Astron., 2, 662
Gupta, R., Mukai, T., Vaidya, D. B., Sen, A. K., \& Okada, Y. 2005, A\&A, 441, 555

Hensley, B. S., \& Draine, B. T. 2017, ApJ, 836, 179

Hensley, B., Murphy, E., \& Staguhn, J. 2015, MNRAS, 449, 809

Hildebrandt, S. R., Rebolo, R., Rubino-Martin, J. A., et al. 2007, MNRAS, 382 594

Hoang, T., Draine, B. T., \& Lazarian, A. 2010, ApJ, 715, 1462

Hoang, T., Lazarian, A., \& Draine, B. T. 2011, ApJ, 741, 87

Hoang, T., Vinh, N.-A., \& Lan, N. Q. 2016, ApJ, 824, 18

Hoang, T., Lan, N.-Q., Vinh, N.-A., \& Kim, Y.-J. 2018, ApJ, 862, 116

Jones, A. P. 2009, A\&A, 506, 797

Kessler-Silacci, J., Augereau, J.-c., Dullemond, C. P., et al. 2006, ApJ, 639, 275

Kisiel, Z. 2001, Spectroscopy from Space (Dordrecht: Springer Netherlands), 91

Kisiel, Z. 2019, PROSPE - Programs for Rotational SPEctroscopy

Krasnokutski, S. A., Rouillé, G., Jäger, C., et al. 2014, ApJ, 782, 15

Lamiel-Garcia, O., Ko, K. C., Lee, J. Y., Bromley, S. T., \& Illas, F. 2017, J. Chem. Theory Comput., 13, 1785

Li, A., \& Draine, B. T. 2001, ApJ, 550, L213

Molster, F. J., Waters, L. B. F. M., Tielens, A. G. G. M., \& Barlow, M. J. 2002, A\&A, 382, 222

Pickett, H., Poynter, R., Cohen, E., et al. 1998, J. Quant. Spectr. Rad. Transf., 60, 883

Purcell, E. M. 1975, The Dusty Universe (New York: Neale Watson Academic Publications), 155

Puzzarini, C., Stanton, John. F., Gauss, J., et al. 2010, Int. Rev. Phys. Chem., 29, 273

Rafikov, R. R. 2006, ApJ, 646, 288

Tibbs, C., Flagey, N., Paladini, R., et al. 2011, MNRAS, 418, 1889

Ubach, C., Maddison, S. T., Wright, C. M., et al. 2017, MNRAS, 466, 4083

Vidal, M., Casassus, S., Dickinson, C., et al. 2011, MNRAS, 414, 2424

Waelkens, C., Waters, L. B. F. M., de Graauw, M. S., et al. 1996, A\&A, 315, 245

Watson, R. A., Rebolo, R., Rubiño-Martín, J. A., et al. 2005, ApJ, 624, L89

Weingartner, J. C., \& Draine, B. T. 2001a, ApJS, 134, 263

Weingartner, J. C., \& Draine, B. T. 2001b, ApJ, 548, 296

Ysard, N., \& Verstraete, L. 2010, A\&A, 509, A12

Zamirri, L., Macià Escatllar, A., Mariñoso Guiu, J., Ugliengo, P., \& Bromley, S. T. 2019, ACS Earth Space Chem., 3, 2323

Zhan, C.-G., Nichols, J. A., \& Dixon, D. A. 2003, J. Phys. Chem. A, 107, 4184 


\section{Appendix A: Rotational emission for a PAH: comparison with other models}

Using the equations described in the Sect. 2, in this appendix we compare the results of our model with those from the works of Ali-Haïmoud et al. (2009) and Draine \& Lazarian (1998). The parameters of each environment can be found in Draine \& Lazarian (1998), where we have used the dust grain temperature as the rotational temperature of our grains. The number of grains for our particle containing $N_{\mathrm{C}}=20$ atoms is extracted from the FORTRAN code in Weingartner \& Draine (2001b) using a radius of $\approx 3.5$. Dust particles of this size are likely to be the most important contributors to the AME, although larger particles also contribute to the emission. Figure A.1 shows a comparison of the results between our model and the results from Draine \& Lazarian (1998) and Ali-Haïmoud et al. (2009). In CNM conditions, where the densities are extremely low and there is a large discrepancy between dust and gas temperature $\left(T_{\mathrm{d}}=20 \mathrm{~K}, T_{\mathrm{g}}=100 \mathrm{~K}\right)$ we find a large discrepancy between published models and our model. Here our calculated emissivities are larger than expected and can not be reconciled by lowering the rotational temperature. For the $\mathrm{MC}$ environment, our result is 1.4 times larger than other published models. Surprisingly, for DC our model provides lower emissivities than those previously estimated. In DC, the densities are sufficiently large to expect a distribution dominated by collision interactions, which would cause the rotational temperature to be equal to the gas kinetic temperature. For $\mathrm{RN}$, while $T_{\mathrm{d}} \neq T_{\mathrm{g}}$, the emissivity is reduced by $60 \%$ compared to other models.

From Fig. A.1 both the emissivity and peak position obtained in our model is in good agreement for the MC, DC and RN cases. Taking into account that the reported data from DL98 and AHHD correspond to total microwave emissivities for all PAHs and our data corresponds to a single particle, it is expected that our results should appear close to or below the reported data points in terms of emissivity, and at slightly larger frequencies in terms of peak position. The MC case shows larger emissivity in our model than expected, while the best case scenarios correspond to $\mathrm{DC}$ and $\mathrm{RN}$ conditions.
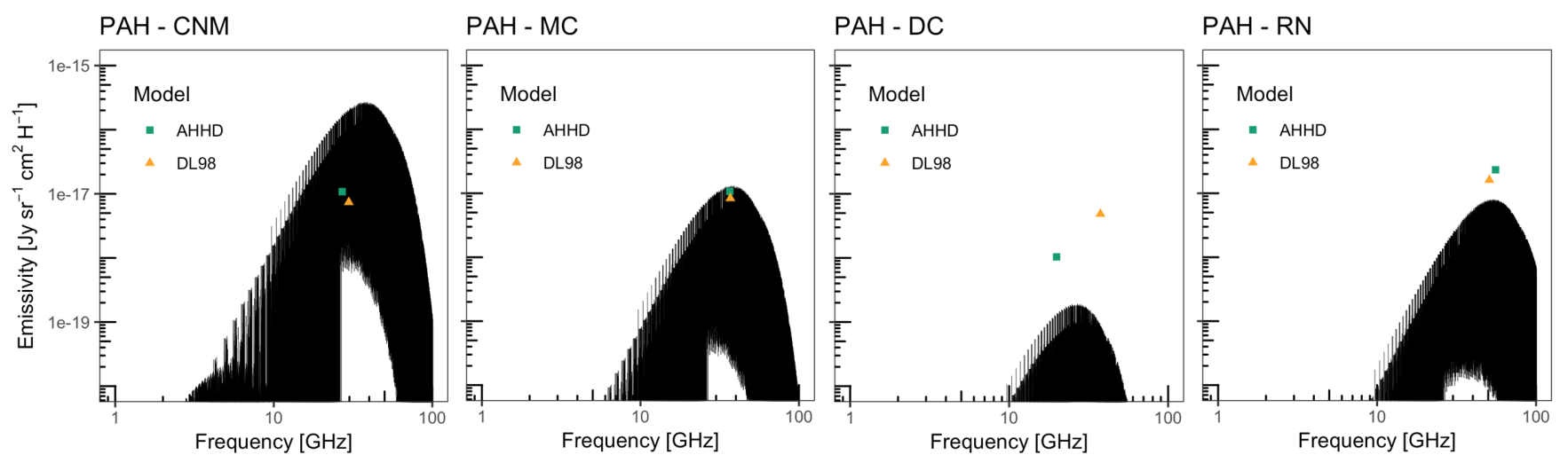

Fig. A.1. Microwave emission from fictitious PAH with the rotational constants $A=432 B=370$ and $C=344 \mathrm{MHz}$ under several astronomical environments: Cold Neutral Medium, Molecular Cloud, Dark Cloud and Reflection Nebula. 
Appendix B: Rotational constants and dipole moments of silicate nanoclusters

Table B.1. Rotational $\left(B_{\alpha}\right)$ constants $(\mathrm{MHz})$ for the nanoclusters in this work.

\begin{tabular}{lrrr}
\hline \hline Molecule & $B_{x}(\mathrm{MHz})$ & $B_{y}(\mathrm{MHz})$ & $B_{z}(\mathrm{MHz})$ \\
\hline $\mathrm{O}-1$ & 3260.983 & 1291.755 & 925.244 \\
$\mathrm{O}-2$ & 614.676 & 583.865 & 373.861 \\
$\mathrm{O}-3$ & 312.054 & 277.278 & 221.305 \\
$\mathrm{O}-4$ & 196.153 & 143.527 & 106.91 \\
$\mathrm{O}-5$ & 114.285 & 101.138 & 75.222 \\
$\mathrm{O}-6$ & 85.73 & 72.891 & 64.873 \\
$\mathrm{O}-7$ & 73.928 & 47.551 & 42.208 \\
$\mathrm{O}-8$ & 54.536 & 40.455 & 38.061 \\
$\mathrm{O}-9$ & 45.888 & 31.399 & 29.14 \\
$\mathrm{O}-10$ & 37.203 & 28.814 & 24.862 \\
$\mathrm{P}-1$ & 9876.871 & 2947.067 & 2269.802 \\
$\mathrm{P}-2$ & 1472.618 & 1176.786 & 746.653 \\
$\mathrm{P}-3$ & 565.57 & 492.799 & 398.548 \\
$\mathrm{P}-4$ & 391.274 & 210.07 & 189.036 \\
$\mathrm{P}-5$ & 279.393 & 158.611 & 146.333 \\
$\mathrm{P}-6$ & 201.405 & 101.628 & 98.244 \\
$\mathrm{P}-7$ & 137.455 & 86.734 & 76.038 \\
$\mathrm{P}-8$ & 94.832 & 82.928 & 62.082 \\
$\mathrm{P}-9$ & 71.393 & 66.017 & 58.03 \\
$\mathrm{P}-10$ & 64.729 & 57.262 & 46.684 \\
$\mathrm{SiO}-2$ & 11267.036 & 5809.04 & 3832.887 \\
$\mathrm{SiO}-3$ & 2590.916 & 2590.65 & 1295.392 \\
$\mathrm{SiO}-4$ & 1224.702 & 1209.03 & 680.397 \\
$\mathrm{SiO}-5$ & 1408.354 & 430.413 & 416.801 \\
$\mathrm{SiO}-6$ & 614.419 & 445.02 & 442.944 \\
$\mathrm{SiO}-7$ & 467.093 & 467.05 & 277.431 \\
$\mathrm{SiO}-8$ & 352.59 & 336.434 & 267.523 \\
$\mathrm{SiO}-9$ & 289.786 & 227.942 & 214.823 \\
$\mathrm{SiO}-10$ & 229.872 & 178.145 & 134.59 \\
$\mathrm{SiO}-11$ & 242.421 & 98.354 & 89.432 \\
$\mathrm{SiO}-12$ & 196.505 & 101.967 & 87.743 \\
$\mathrm{SiO}-13$ & 155.539 & 82.323 & 76.655 \\
$\mathrm{SiO}-14$ & 148.656 & 64.472 & 64.22 \\
$\mathrm{SiO}-15$ & 133.012 & 52.728 & 50.781 \\
$\mathrm{SiO}-16$ & 158.38 & 48.654 & 46.506 \\
$\mathrm{SiO}-17$ & 81.492 & 68.293 & 51.566 \\
$\mathrm{SiO}-18$ & 90.666 & 42.475 & 41.509 \\
$\mathrm{SiO}-19$ & 86.865 & 50.438 & 50.425 \\
$\mathrm{SiO}-20$ & 72.194 & 46.981 & 42.23 \\
\hline & & &
\end{tabular}

Table B.2. Total dipole moments $(\mu)$ in Debyes $(D)$ for olivine $(\mathrm{O}-N)$, pyroxene $(\mathrm{P}-N)$ and silicon monoxide $(\mathrm{SiO}-N)$ nanoclusters.

\begin{tabular}{lr}
\hline \hline Molecule & $\mu(\mathrm{D})$ \\
\hline $\mathrm{O}-1$ & 9.50 \\
$\mathrm{O}-2$ & 6.00 \\
$\mathrm{O}-3$ & 1.99 \\
$\mathrm{O}-4$ & 11.60 \\
$\mathrm{O}-5$ & 2.03 \\
$\mathrm{O}-6$ & 3.28 \\
$\mathrm{O}-7$ & 6.60 \\
$\mathrm{O}-8$ & 6.56 \\
$\mathrm{O}-9$ & 5.79 \\
$\mathrm{O}-10$ & 3.27 \\
$\mathrm{P}-1$ & 12.60 \\
$\mathrm{P}-2$ & 0.00 \\
$\mathrm{P}-3$ & 3.28 \\
$\mathrm{P}-4$ & 2.83 \\
$\mathrm{P}-5$ & 4.28 \\
$\mathrm{P}-6$ & 0.00 \\
$\mathrm{P}-7$ & 6.39 \\
$\mathrm{P}-8$ & 5.29 \\
$\mathrm{P}-9$ & 6.61 \\
$\mathrm{P}-10$ & 5.29 \\
$\mathrm{SiO}-2$ & 0.00 \\
$\mathrm{SiO}-3$ & 0.00 \\
$\mathrm{SiO}-4$ & 0.00 \\
$\mathrm{SiO}-5$ & 0.73 \\
$\mathrm{SiO}-6$ & 2.20 \\
$\mathrm{SiO}-7$ & 0.18 \\
$\mathrm{SiO}-8$ & 1.68 \\
$\mathrm{SiO}-9$ & 1.79 \\
$\mathrm{SiO}-10$ & 1.41 \\
$\mathrm{SiO}-11$ & 1.19 \\
$\mathrm{SiO}-12$ & 0.92 \\
$\mathrm{SiO}-13$ & 1.44 \\
$\mathrm{SiO}-14$ & 1.08 \\
$\mathrm{SiO}-15$ & 1.52 \\
$\mathrm{SiO}-16$ & 0.76 \\
$\mathrm{SiO}-17$ & 1.63 \\
$\mathrm{SiO}-18$ & 1.78 \\
$\mathrm{SiO}-19$ & 3.13 \\
$\mathrm{SiO}-20$ & 3.55 \\
\hline &
\end{tabular}




\section{Appendix C: Estimate of changes due to centrifugal distortion}

The frequencies at which a linear rotor would emit are calculated as:

$v_{R}(J)=2 B(J+1)-4 D(J+1)^{3}$.

In general, the difference in orders of magnitude between $B$ and $D$ is of the order of 4 to 6 . As stated in the text, the Gaussian 16 package can calculate centrifugal constants. In Table C.1 we compare the smallest rotational constant $\left(B_{z}\right)$ with the centrifugal constants $\left(D_{j}\right)$ for P-1 and P-2 nanoclusters.

The difference in orders of magnitude are 6 for P-1 and 7 for P-2. We can estimate the importance of the centrifugal term by calculating the fraction $4 D(J+1)^{3} / 2 B(J+1)$ using $J=400$, $B=1$ and two values of $D_{1}=10^{-6}$ and $D_{2}=10^{-7}$. For $D_{1}$, the fraction is 0.32 , which means that the frequencies can be shifted up to $32 \%$, while for $D_{2}$, the fraction lowers down to $3.2 \%$.
Table C.1. Rotational (MHz) and Wilson centrifugal distortion constants (kHZ) for P-1 and P-2 nanoclusters.

\begin{tabular}{lrrrr}
\hline \hline Molecule & $B_{z}(\mathrm{MHz})$ & $D_{j}(\mathrm{kHz})$ & $D_{j} k(\mathrm{kHz})$ & $D_{k}(\mathrm{kHz})$ \\
\hline $\mathrm{P}-1$ & 2269.802 & 0.4145 & 1.7794 & 6.3816 \\
$\mathrm{P}-2$ & 746.653 & 0.0531 & -0.102 & 0.0357 \\
\hline
\end{tabular}

We remind the reader that $J=400$ may only be accessible for the largest O-10 nanocluster, while the smallest particles should achieve maximum $J$ values of the order of $\sim 50$ to $\sim 100$. For the P-1 nanocluster, we find that using the reported data in Table C.1 the difference is of the order of $0.2 \%$. The centrifugal distortion term will likely reduce as size increases, thus the most likely value for the O-10 is $D_{2}$ rather than $D_{1}$. Hence, we expect our results to have a typical error of approximately $3 \%$ with respect to the real spectra. 